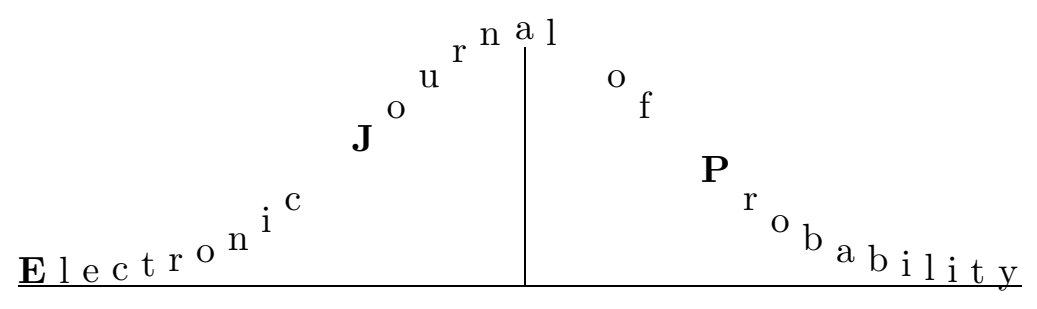

Vol. 11 (2006), Paper no. 7, pages 162-198.

Journal URL

http://www.math.washington.edu/ ejpecp/

\title{
Brownian local minima, random dense countable sets and random equivalence classes
}

\author{
Boris Tsirelson \\ School of Mathematics \\ Tel Aviv University \\ Tel Aviv 69978, Israel \\ tsirel@post.tau.ac.il \\ http://www.tau.ac.il/ ${ }^{\sim}$ tsirel/
}

\begin{abstract}
A random dense countable set is characterized (in distribution) by independence and stationarity. Two examples are Brownian local minima and unordered infinite sample. They are identically distributed. A framework for such concepts, proposed here, includes a wide class of random equivalence classes.
\end{abstract}

Key words: Brownian motion, local minimum, point process, equivalence relation.

AMS 2000 Subject Classification: Primary 60J65; Secondary 60B99, 60D05, $60 \mathrm{G} 55$.

Submitted to EJP on January 29, 2006. Final version accepted on February 27, $2006 .^{1}$

\footnotetext{
${ }^{1}$ This research was supported by THE ISRAEL SCIENCE FOUNDATION (grant No. 683/05).
} 


\section{Introduction}

Random dense countable sets arise naturally from the Brownian motion (local extrema, see [5, 2.9.12]), percolation (double, or four-arm points, see [2]), oriented percolation (points of type $(2,1)$, see [3, Th. 5.15]) etc. They are scarcely investigated, because they fail to fit into the usual framework. They cannot be treated as random elements of 'good' (Polish, standard) spaces. The framework of adapted Poisson processes, used by Aldous and Barlow [1], does not apply to the Brownian motion, since the latter cannot be correlated with a Poisson process adapted to the same filtration. The 'hit-and-miss' framework used by Kingman [10] and Kendall [9] fails to discern the clear-cut distinction between Brownian local minima and, say, randomly shifted set of rational numbers. A new approach introduced here catches this distinction, does not use adapted processes, and shows that Brownian local minima are distributed like an infinite sample in the following sense (see Theorem 6.11).

Theorem. There exists a probability measure $P$ on the product space $C[0,1] \times(0,1)^{\infty}$ such that

(a) the first marginal of $P$ (that is, projection to the first factor) is the Wiener measure on the space $C[0,1]$ of continuous paths $w:[0,1] \rightarrow \mathbb{R}$;

(b) the second marginal of $P$ is the Lebesgue measure on the cube $(0,1)^{\infty}$ of infinite (countable) dimension;

(c) P-almost all pairs $(w, u), w \in C[0,1], u=\left(u_{1}, u_{2}, \ldots\right) \in(0,1)^{\infty}$, are such that the numbers $u_{1}, u_{2}, \ldots$ are an enumeration of the set of all local minimizers of the Brownian path $w$.

Thus, the conditional distribution of $u_{1}, u_{2}, \ldots$ given $w$ provides a (randomized) enumeration of Brownian minimizers by independent uniform random variables.

The same result holds for every random dense countable set that satisfies conditions of independence and stationarity, see Definitions 4.2, 6.8 and Theorem 6.9. Two-dimensional generalizations, covering the percolation-related models, are possible.

On a more abstract level the new approach is formalized in Sections 7, 8 in the form of 'borelogy' that combines some ideas of descriptive set theory [6] and diffeology [4]. Random elements of various quotient spaces fit into the new framework. Readers that like abstract concepts may start with these sections. 


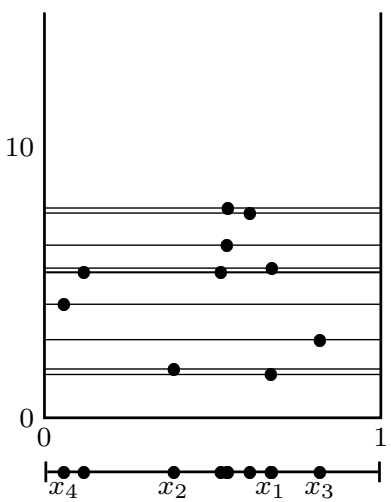

(a)

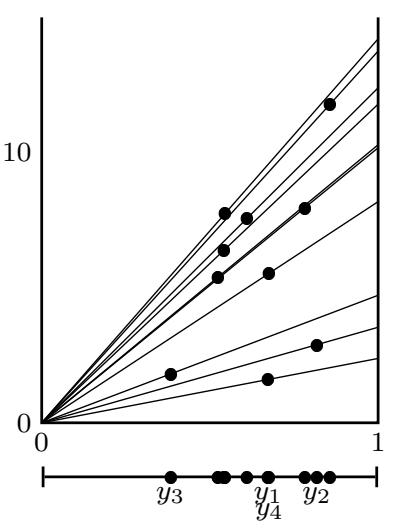

(b)

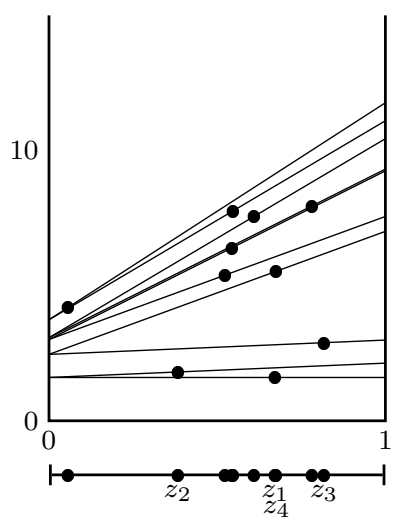

(c)

Figure 1: Different enumerations turn the same set into an infinite sample from: (a) the uniform distribution, (b) the triangular distribution, (c) their mixture (uniform $z_{1}, z_{3}, \ldots$ but triangular $\left.z_{2}, z_{4}, \ldots\right)$.

\section{Main lemma}

Before the main lemma we consider an instructive special case.

1.1 Example. Let $\mu$ be the uniform distribution on the interval $(0,1)$ and $\nu$ the triangle distribution on the same interval, that is,

$$
\mu(B)=\int_{B} \mathrm{~d} x, \quad \nu(B)=\int_{B} 2 x \mathrm{~d} x
$$

for all Borel sets $B \subset(0,1)$. On the space $(0,1)^{\infty}$ of sequences, the product measure $\mu^{\infty}$ is the joint distribution of uniform i.i.d. random variables, while $\nu^{\infty}$ is the joint distribution of triangular i.i.d. random variables. I claim existence of a probability measure $P$ on $(0,1)^{\infty} \times(0,1)^{\infty}$ such that

(a) the first marginal of $P$ is equal to $\mu^{\infty}$ (that is, $P\left(B \times(0,1)^{\infty}\right)=\mu^{\infty}(B)$ for all Borel sets $\left.B \subset(0,1)^{\infty}\right)$;

(b) the second marginal of $P$ is equal to $\nu^{\infty}$ (that is, $P\left((0,1)^{\infty} \times B\right)=\nu^{\infty}(B)$ for all Borel sets $\left.B \subset(0,1)^{\infty}\right)$;

(c) $P$-almost all pairs $(x, y), x=\left(x_{1}, x_{2}, \ldots\right) \in(0,1)^{\infty}, y=\left(y_{1}, y_{2}, \ldots\right) \in(0,1)^{\infty}$ are such that

$$
\left\{x_{1}, x_{2}, \ldots\right\}=\left\{y_{1}, y_{2}, \ldots\right\}
$$

in other words, the sequence $y$ is a permutation of the sequence $x$. (A random permutation, of course.)

A paradox: the numbers $y_{k}$ are biased toward 1, the numbers $x_{k}$ are not; nevertheless they are just the same numbers! 
An explanation (and a sketchy proof) is shown on Fig. 1(a,b). A countable subset of the strip $(0,1) \times(0, \infty)$ is a realization of a Poisson point process. (The mean number of points in any domain is equal to its area.) The first 10 points of the same countable set are shown on both figures, but on Fig. 1(a) the points are ordered according to the vertical coordinate, while on Fig. 1(b) they are ordered according to the ratio of the two coordinates. We observe that $\left\{y_{1}, \ldots, y_{10}\right\}$ is indeed biased toward 1 , while $\left\{x_{1}, \ldots, x_{10}\right\}$ is not. On the other hand, $y$ is a permutation of $x$. (This time, $y_{1}=x_{1}, y_{2}=x_{3}, y_{3}=x_{2}$, $\ldots)$

A bit more complicated ordering, shown on Fig. 1(c), serves the measure $\mu \times \nu \times \mu \times \nu \times \ldots$, the joint distribution of independent, differently distributed random variables.

In every case we use an increasing sequence of (random) functions $h_{n}:(0,1) \rightarrow[0, \infty)$ such that for each $n$ the graph of $h_{n}$ contains a Poisson point, while the region between the graphs of $h_{n-1}$ and $h_{n}$ does not. The differences $h_{n}-h_{n-1}$ are constant functions on Fig. 1(a), triangular (that is, $x \mapsto$ const $\cdot x$ ) on Fig. 1(b), while on Fig. 1(c) they are constant for odd $n$ and triangular for even $n$.

Moreover, the same idea works for dependent random variables. In this case $h_{n}-h_{n-1}$ is proportional to the conditional density, given the previous points. We only need existence of conditional densities and divergence of their sum (in order to exhaust the strip).

Here is the main lemma.

1.2 Lemma. Let $\mu$ be a probability measure on $(0,1)^{\infty}$ such that

(a) for every $n$ the marginal distribution of the first $n$ coordinates is absolutely continuous;

(b) for almost all $x \in(0,1)$ and $\mu$-almost all $\left(x_{1}, x_{2}, \ldots\right) \in(0,1)^{\infty}$,

$$
\sum_{n=1}^{\infty} \frac{f_{n+1}\left(x_{1}, \ldots, x_{n}, x\right)}{f_{n}\left(x_{1}, \ldots, x_{n}\right)}=\infty
$$

here $f_{n}$ is the density of the first $n$ coordinates.

Let $\nu$ be another probability measure on $(0,1)^{\infty}$ satisfying the same conditions (a), (b). Then there exists a probability measure $P$ on $(0,1)^{\infty} \times(0,1)^{\infty}$ such that

(c) the first marginal of $P$ is equal to $\mu$;

(d) the second marginal of $P$ is equal to $\nu$;

(e) P-almost all pairs $(x, y), x=\left(x_{1}, x_{2}, \ldots\right) \in(0,1)^{\infty}, y=\left(y_{1}, y_{2}, \ldots\right) \in(0,1)^{\infty}$ are such that

$$
\left\{x_{1}, x_{2}, \ldots\right\}=\left\{y_{1}, y_{2}, \ldots\right\}
$$

In other words, the sequence $y$ is a permutation of the sequence $x$ (since $x_{k}$ are pairwise different due to absolute continuity, as well as $y_{k}$ ).

The rest of the section is occupied by the proof of the main lemma. 
Throughout the proof, Poisson point processes on the strip $(0,1) \times[0, \infty)$ (or its measurable part) are such that the mean number of points in any measurable subset is equal to its two-dimensional Lebesgue measure. Random variables (and random functions) are treated here as measurable functions of the original Poisson point process on the strip.

We start with three rather general claims.

1.3 Claim. (a) A Poisson point process on the strip $(0,1) \times[0, \infty)$ may be treated as the set $\Pi$ of random points $\left(U_{n}, T_{1}+\cdots+T_{n}\right)$ for $n=1,2, \ldots$, where $U_{1}, T_{1}, U_{2}, T_{2}, \ldots$ are independent random variables, each $U_{k}$ is distributed uniformly on $(0,1)$, and each $T_{k}$ is distributed $\operatorname{Exp}(1)$ (that is, $\mathbb{P}\left(T_{k}>t\right)=\mathrm{e}^{-t}$ for $\left.t \geq 0\right)$;

(b) conditionally on $\left(U_{1}, T_{1}\right)$, the set $\Pi_{1}=\left\{\left(U_{n}, T_{1}+\cdots+T_{n}\right): n \geq 2\right\}$ is (distributed as) a Poisson point process on $(0,1) \times\left[T_{1}, \infty\right)$.

The proof is left to the reader.

1.4 Claim. Let $f:(0,1) \rightarrow[0, \infty)$ be a measurable function satisfying $\int_{0}^{1} f(x) \mathrm{d} x=1$, and $\Pi$ be a Poisson point process on $(0,1) \times[0, \infty)$. Then

(a) the minimum

$$
t_{1}=\min _{(x, y) \in \Pi} \frac{y}{f(x)}
$$

(where $y / 0=\infty)$ is reached at a single point $\left(x_{1}, y_{1}\right) \in \Pi$;

(b) $x_{1}$ and $t_{1}=y_{1} / f\left(x_{1}\right)$ are independent, $t_{1}$ is distributed $\operatorname{Exp}(1)$, and the distribution of $x_{1}$ has the density $f$;

(c) conditionally on $\left(x_{1}, y_{1}\right)$, the set $\Pi_{1}=\Pi \backslash\left\{\left(x_{1}, y_{1}\right)\right\}$ is (distributed as) a Poisson point process on $\left\{(x, y): 0<x<1, t_{1} f(x)<y<\infty\right\}$.

Proof. A special case, $f(x)=1$ for all $x$, follows from 1.3 .

A more general case, $f(x)>0$ for all $x$, results from the special case by the transformation $(x, y) \mapsto(F(x), y / f(x))$ where $F(x)=\int_{0}^{x} f\left(x^{\prime}\right) \mathrm{d} x^{\prime}$. The transformation preserves Lebesgue measure on $(0,1) \times[0, \infty)$, therefore it preserves also the Poisson point process.

In the general case the same transformation sends $A \times[0, \infty)$ to $(0,1) \times[0, \infty)$, where $A=\{x: f(x)>0\}$. Conditionally on $\left(x_{1}, t_{1}\right)$ we get a Poisson point process on $\{(x, y)$ : $\left.x \in A, t_{1} f(x)<y<\infty\right\}$ independent of the Poisson point process on $\{(x, y): x \notin A, 0<$ $y<\infty\}$.

1.5 Claim. Let $f:(0,1) \rightarrow[0, \infty)$ and $g:(0,1) \times(0,1) \rightarrow[0, \infty)$ be measurable functions satisfying $\int_{0}^{1} f(x) \mathrm{d} x=1$ and $\int_{0}^{1} g\left(x_{1}, x_{2}\right) \mathrm{d} x_{2}=1$ for almost all $x_{1}$. Let $\Pi$ be a Poisson point process on $(0,1) \times[0, \infty)$, while $\left(x_{1}, y_{1}\right), t_{1}$ and $\Pi_{1}$ be as in 1.4 . Then

(a) the minimum

$$
t_{2}=\min _{(x, y) \in \Pi_{1}} \frac{y-t_{1} f(x)}{g\left(x_{1}, x\right)}
$$

is reached at a single point $\left(x_{2}, y_{2}\right) \in \Pi_{1}$; 
(b) conditionally on $\left(x_{1}, y_{1}\right)$ we have: $x_{2}$ and $t_{2}=\left(y_{2}-t_{1} f\left(x_{2}\right)\right) / g\left(x_{1}, x_{2}\right)$ are independent, $t_{2}$ is distributed $\operatorname{Exp}(1)$, and the distribution of $x_{2}$ has the density $g\left(x_{1}, \cdot\right)$ (conditional distributions are meant);

(c) conditionally on $\left(x_{1}, y_{1}\right),\left(x_{2}, y_{2}\right)$, the set $\Pi_{2}=\Pi \backslash\left\{\left(x_{1}, t_{1}\right),\left(x_{2}, y_{2}\right)\right\}$ is a Poisson point process on $\left\{(x, y): 0<x<1, t_{1} f(x)+t_{2} g\left(x_{1}, x\right)<y<\infty\right\}$.

Proof. After conditioning on $\left(x_{1}, y_{1}\right)$ we apply 1.4 to the Poisson point process $\{(x, y-$ $\left.\left.t_{1} f(x)\right):(x, y) \in \Pi_{1}\right\}$ on $(0,1) \times[0, \infty)$ and the function $g\left(x_{1}, \cdot\right):(0,1) \rightarrow[0, \infty)$.

Equipped with these claims we prove the main lemma as follows. Introducing conditional densities

$$
g_{n+1}\left(x \mid x_{1}, \ldots, x_{n}\right)=\frac{f_{n+1}\left(x_{1}, \ldots, x_{n}, x\right)}{f_{n}\left(x_{1}, \ldots, x_{n}\right)}, \quad g_{1}(x)=f_{1}(x)
$$

and a Poisson point process $\Pi$ on the strip $(0,1) \times[0, \infty)$, we construct a sequence of points $\left(X_{n}, Y_{n}\right)$ of $\Pi$, random variables $T_{n}$ and random functions $H_{n}$ as follows:

$$
\begin{aligned}
T_{1}= & \min _{(x, y) \in \Pi} \frac{y}{g_{1}(x)}=\frac{Y_{1}}{g_{1}\left(X_{1}\right)} ; \\
H_{1}(x)= & T_{1} g_{1}(x) ; \\
T_{2}= & \min _{(x, y) \in \Pi_{1}} \frac{y-H_{1}(x)}{g_{2}\left(x \mid X_{1}\right)}=\frac{Y_{2}-H_{1}\left(X_{2}\right)}{g_{2}\left(X_{2} \mid X_{1}\right)} ; \\
H_{2}(x)= & H_{1}(x)+T_{2} g_{2}\left(x \mid X_{1}\right) ; \\
& \ldots \\
T_{n}= & \min _{(x, y) \in \Pi_{n-1}} \frac{y-H_{n-1}(x)}{g_{n}\left(x \mid X_{1}, \ldots, X_{n-1}\right)}=\frac{Y_{n}-H_{n-1}\left(X_{n}\right)}{g_{n}\left(X_{n} \mid X_{1}, \ldots, X_{n-1}\right)} ; \\
H_{n}(x)= & H_{n-1}(x)+T_{n} g_{n}\left(x \mid X_{1}, \ldots, X_{n-1}\right) ;
\end{aligned}
$$

here $\Pi_{n}$ stands for $\Pi \backslash\left\{\left(X_{1}, Y_{1}\right), \ldots,\left(X_{n}, Y_{n}\right)\right\}$. By $1.4(\mathrm{~b}), X_{1}$ and $T_{1}$ are independent, $T_{1} \sim \operatorname{Exp}(1)$ and $X_{1} \sim g_{1}$. By 1.4(c) and 1.5(b), conditionally on $X_{1}$ and $T_{1}, \Pi_{1}$ is a Poisson point process on $\left\{(x, y): y>H_{1}(x)\right\}$, while $X_{2}$ and $T_{2}$ are independent, $T_{2} \sim \operatorname{Exp}(1)$ and $X_{2} \sim g_{2}\left(\cdot \mid X_{1}\right)$. It follows that $T_{1}, T_{2}$ and $\left(X_{1}, X_{2}\right)$ are independent, and the joint distribution of $X_{1}, X_{2}$ has the density $g_{1}\left(x_{1}\right) g_{2}\left(x_{2} \mid x_{1}\right)=f_{2}\left(x_{1}, x_{2}\right)$. By $1.5(\mathrm{c})$, $\Pi_{2}$ is a Poisson point process on $\left\{(x, y): y>H_{2}(x)\right\}$ conditionally, given $\left(X_{1}, Y_{1}\right)$ and $\left(X_{2}, Y_{2}\right)$.

The same arguments apply for any $n$. We get two independent sequences, $\left(T_{1}, T_{2}, \ldots\right)$ and $\left(X_{1}, X_{2}, \ldots\right)$. Random variables $T_{n}$ are independent, distributed $\operatorname{Exp}(1)$ each. The joint distribution of $X_{1}, X_{2}, \ldots$ is equal to $\mu$, since for every $n$ the joint distribution of $X_{1}, \ldots, X_{n}$ has the density $f_{n}$. Also, $\Pi_{n}$ is a Poisson point process on $\left\{(x, y): y>H_{n}(x)\right\}$ conditionally, given $\left(X_{1}, Y_{1}\right), \ldots,\left(X_{n}, Y_{n}\right)$.

1.6 Claim. $H_{n}(x) \uparrow \infty$ for almost all pairs $(\Pi, x)$. 
Proof. By $1.2(\mathrm{~b}), \sum_{n} g_{n+1}\left(x \mid x_{1}, \ldots, x_{n}\right)=\infty$ for almost all $x \in(0,1)$ and $\mu$-almost all $\left(x_{1}, x_{2}, \ldots\right)$. Therefore $\sum_{n} g_{n+1}\left(x \mid X_{1}, \ldots, X_{n}\right)=\infty$ for almost all $x \in(0,1)$ and almost all $\Pi$. It is easy to see that $\sum_{n} c_{n} T_{n}=\infty$ a.s. for each sequence $\left(c_{n}\right)_{n}$ such that $\sum_{n} c_{n}=\infty$. Taking into account that $\left(T_{1}, T_{2}, \ldots\right)$ is independent of $\left(X_{1}, X_{2}, \ldots\right)$ we conclude that $\sum_{n} T_{n} g_{n}\left(x \mid X_{1}, \ldots, X_{n-1}\right)=\infty$ for almost all $x$ and $\Pi$. The partial sums of this series are nothing but $H_{n}(x)$.

Still, we have to prove that the points $\left(X_{n}, Y_{n}\right)$ exhaust the set $\Pi$. Of course, a non-random negligible set does not intersect $\Pi$ a.s.; however, the negligible set $\left\{x: \lim _{n} H_{n}(x)<\infty\right\}$ is random.

1.7 Claim. The set $\cap_{n} \Pi_{n}$ is empty a.s.

Proof. It is sufficient to prove that $\cap_{n} \Pi_{n, M}=\emptyset$ a.s. for every $M \in(0, \infty)$, where $\Pi_{n, M}=$ $\left\{(x, y) \in \Pi_{n}: y<M\right\}$. Conditionally, given $\left(X_{1}, Y_{1}\right), \ldots,\left(X_{n}, Y_{n}\right)$, the set $\Pi_{n, M}$ is a Poisson point process on $\left\{(x, y): H_{n}(x)<y<M\right\}$; the number $\left|\Pi_{n, M}\right|$ of points in $\Pi_{n, M}$ satisfies

$$
\mathbb{E}\left(\left|\Pi_{n, M}\right| \mid\left(X_{1}, Y_{1}\right), \ldots,\left(X_{n}, Y_{n}\right)\right)=\int_{0}^{1}\left(M-H_{n}(x)\right)^{+} \mathrm{d} x .
$$

Therefore

$$
\mathbb{E}\left|\Pi_{n, M}\right|=\mathbb{E} \int_{0}^{1}\left(M-H_{n}(x)\right)^{+} \mathrm{d} x .
$$

By 1.6 and the monotone convergence theorem, $\mathbb{E} \int_{0}^{1}\left(M-H_{n}(x)\right)^{+} \mathrm{d} x \rightarrow 0$ as $n \rightarrow \infty$. Thus, $\lim _{n}\left|H_{n, M}\right|=0$ a.s.

Now we are in position to finish the proof of the main lemma. Applying our construction twice (for $\mu$ and for $\nu$ ) we get two enumerations of a single Poisson point process on the strip,

$$
\left\{\left(X_{n}, Y_{n}\right): n=1,2, \ldots\right\}=\Pi=\left\{\left(X_{n}^{\prime}, Y_{n}^{\prime}\right): n=1,2, \ldots\right\},
$$

such that the sequence $\left(X_{1}, X_{2}, \ldots\right)$ is distributed $\mu$ and the sequence $\left(X_{1}^{\prime}, X_{2}^{\prime}, \ldots\right)$ is distributed $\nu$. The joint distribution $P$ of these two sequences satisfies the conditions $1.2(\mathrm{c}, \mathrm{d}, \mathrm{e})$.

\section{Random countable sets}

Following the 'constructive countability' approach of Kendall [9, Def. 3.3] we treat a random countable subset of $(0,1)$ as

$$
\omega \mapsto\left\{X_{1}(\omega), X_{2}(\omega), \ldots\right\}
$$

where $X_{1}, X_{2}, \cdots: \Omega \rightarrow(0,1)$ are random variables. (To be exact, it would be called a random finite or countable set, since $X_{n}(\omega)$ need not be pairwise distinct.) It may happen that 
$\left\{X_{1}(\omega), X_{2}(\omega), \ldots\right\}=\left\{Y_{1}(\omega), Y_{2}(\omega), \ldots\right\}$ for almost all $\omega$ (the sets are equal, multiplicity does not matter); then we say that the two sequences $\left(X_{k}\right)_{k},\left(Y_{k}\right)_{k}$ of random variables represent the same random countable set, and write $\left\{X_{1}, X_{2}, \ldots\right\}=\left\{Y_{1}, Y_{2}, \ldots\right\}$. On the other hand it may happen that the joint distribution of $X_{1}, X_{2}, \ldots$ is equal to the joint distribution of some random variables $X_{1}^{\prime}, X_{2}^{\prime}, \cdots: \Omega^{\prime} \rightarrow(0,1)$ on some probability space $\Omega^{\prime}$; then we may say that the two random countable sets $\left\{X_{1}, X_{2}, \ldots\right\},\left\{X_{1}^{\prime}, X_{2}^{\prime}, \ldots\right\}$ are identically distributed. We combine these two ideas as follows.

2.2 Definition. Two random countable sets $\left\{X_{1}, X_{2}, \ldots\right\},\left\{Y_{1}, Y_{2}, \ldots\right\}$ are identically distributed (in other words, $\left\{Y_{1}, Y_{2}, \ldots\right\}$ is distributed like $\left\{X_{1}, X_{2}, \ldots\right\}$ ), if there exists a probability measure $P$ on the space $(0,1)^{\infty} \times(0,1)^{\infty}$ such that

(a) the first marginal of $P$ is equal to the joint distribution of $X_{1}, X_{2}, \ldots$;

(b) the second marginal of $P$ is equal to the joint distribution of $Y_{1}, Y_{2}, \ldots$;

(c) $P$-almost all pairs $(x, y), x=\left(x_{1}, x_{2}, \ldots\right) \in(0,1)^{\infty}, y=\left(y_{1}, y_{2}, \ldots\right) \in(0,1)^{\infty}$ are such that

$$
\left\{x_{1}, x_{2}, \ldots\right\}=\left\{y_{1}, y_{2}, \ldots\right\}
$$

A sufficient condition is given by Main lemma 1.2: if Conditions (a), (b) of Main lemma are satisfied both by the joint distribution of $X_{1}, X_{2}, \ldots$ and by the joint distribution of $Y_{1}, Y_{2}, \ldots$ then $\left\{X_{1}, X_{2}, \ldots\right\}$ and $\left\{Y_{1}, Y_{2}, \ldots\right\}$ are identically distributed.

2.3 Remark. The relation defined by 2.2 is transitive. Having a joint distribution of two sequences $\left(X_{k}\right)_{k}$ and $\left(Y_{k}\right)_{k}$ and a joint distribution of $\left(Y_{k}\right)_{k}$ and $\left(Z_{k}\right)_{k}$ we may construct an appropriate joint distribution of three sequences $\left(X_{k}\right)_{k},\left(Y_{k}\right)_{k}$ and $\left(Z_{k}\right)_{k}$; for example, $\left(X_{k}\right)_{k}$ and $\left(Z_{k}\right)_{k}$ may be made conditionally independent given $\left(Y_{k}\right)_{k}$.

2.4 Definition. A random countable set $\left\{X_{1}, X_{2}, \ldots\right\}$ has the uniform distribution (in other words, is uniform), if $\left\{X_{1}, X_{2}, \ldots\right\}$ and $\left\{Y_{1}, Y_{2}, \ldots\right\}$ are identically distributed for some (therefore, all) $Y_{1}, Y_{2}, \ldots$ whose joint distribution satisfies Conditions (a), (b) of Main lemma 1.2 .

The joint distribution of $X_{1}, X_{2}, \ldots$ may violate Condition $1.2(\mathrm{~b})$, see 9.8 .

If $X_{1}, X_{2}, \ldots$ are i.i.d. random variables then the random countable set $\left\{X_{1}, X_{2}, \ldots\right\}$ may be called an unordered infinite sample from the corresponding distribution. If the latter has a non-vanishing density on $(0,1)$ then $\left\{X_{1}, X_{2}, \ldots\right\}$ has the uniform distribution. A paradox: the distribution of the sample does not depend on the underlying one-dimensional distribution! (See also 9.9.)

2.5 Remark. If the joint distribution of $X_{1}, X_{2}, \ldots$ satisfies Condition (a) of Main lemma (but may violate (b)) then the random countable set $\left\{X_{1}, X_{2}, \ldots\right\}$ may be treated as a part (subset) of a uniform random countable set, in the following sense.

We say that $\left\{X_{1}, X_{2}, \ldots\right\}$ is distributed as a part of $\left\{Y_{1}, Y_{2}, \ldots\right\}$ if there exists $P$ satisfying (a), (b) of 2.2 and (c) modified by replacing the equality $\left\{x_{1}, x_{2}, \ldots\right\}=\left\{y_{1}, y_{2}, \ldots\right\}$ with the inclusion $\left\{x_{1}, x_{2}, \ldots\right\} \subset\left\{y_{1}, y_{2}, \ldots\right\}$. 
Indeed, the proof of Main lemma uses Condition (b) only for exhausting all elements of the Poisson random set.

\section{Selectors}

A single-element part of a random countable set is of special interest.

3.1 Definition. A selector of a random countable set $\left\{X_{1}, X_{2}, \ldots\right\}$ is a probability measure $P$ on the space $(0,1)^{\infty} \times(0,1)$ such that

(a) the first marginal of $P$ is equal to the joint distribution of $X_{1}, X_{2}, \ldots$;

(b) $P$-almost all pairs $(x, z), x=\left(x_{1}, x_{2}, \ldots\right) \in(0,1)^{\infty}, z \in(0,1)$ satisfy

$$
z \in\left\{x_{1}, x_{2}, \ldots\right\}
$$

The second marginal of $P$ is called the distribution of the selector.

Less formally, a selector is a randomized choice of a single element. The conditional distribution $P_{x}$ of $z$ given $x$ is a probability measure concentrated on $\left\{x_{1}, x_{2}, \ldots\right\}$. This measure may happen to be a single atom, which leads to a non-randomized selector

$$
z=x_{N\left(x_{1}, x_{2}, \ldots\right)},
$$

where $N$ is a Borel map $(0,1)^{\infty} \rightarrow\{1,2, \ldots\}$. (See also 3.9.)

In order to prove existence of selectors with prescribed distributions we use a deep duality theory for measures with given marginals, due to Kellerer. It holds for a wide class of spaces $\mathcal{X}_{1}, \mathcal{X}_{2}$, but we need only two. Below, in 3.4, 3.5 and 3.6 we assume that

$$
\begin{aligned}
& \mathcal{X}_{1} \text { is either }(0,1) \text { or }(0,1)^{\infty}, \\
& \mathcal{X}_{2} \text { is either }(0,1) \text { or }(0,1)^{\infty} .
\end{aligned}
$$

Here is the result used here and once again in Sect. 8 .

3.4 Theorem. (Kellerer) Let $\mu_{1}, \mu_{2}$ be probability measures on $\mathcal{X}_{1}, \mathcal{X}_{2}$ respectively, and $B \subset \mathcal{X}_{1} \times \mathcal{X}_{2}$ a Borel set. Then

$$
S_{\mu_{1}, \mu_{2}}(B)=I_{\mu_{1}, \mu_{2}}(B),
$$

where $S_{\mu_{1}, \mu_{2}}(B)$ is the supremum of $\mu(B)$ over all probability measures $\mu$ on $\mathcal{X}_{1} \times \mathcal{X}_{2}$ with marginals $\mu_{1}, \mu_{2}$, and $I_{\mu_{1}, \mu_{2}}(B)$ is the infimum of $\mu_{1}\left(B_{1}\right)+\mu_{2}\left(B_{2}\right)$ over all Borel sets $B_{1} \subset \mathcal{X}_{1}, B_{2} \subset \mathcal{X}_{2}$ such that $B \subset\left(B_{1} \times \mathcal{X}_{2}\right) \cup\left(\mathcal{X}_{1} \times B_{2}\right)$.

See [8, Corollary 2.18 and Proposition 3.3].

Note that $B$ need not be closed.

In fact, the infimum $I_{\mu_{1}, \mu_{2}}(B)$ is always reached [8, Prop. 3.5], but the supremum $S_{\mu_{1}, \mu_{2}}(B)$ is not always reached [8, Example 2.20]. 
3.5 Remark. (a) If $\mu_{1}, \mu_{2}$ are positive (not just probability) measures such that $\mu_{1}\left(\mathcal{X}_{1}\right)=$ $\mu_{2}\left(\mathcal{X}_{2}\right)$ then still $S_{\mu_{1}, \mu_{2}}(B)=I_{\mu_{1}, \mu_{2}}(B)$.

(b) $S_{\mu_{1}, \mu_{2}}(B)$ is equal to the supremum of $\nu(B)$ over all positive (not just probability) measures $\nu$ on $B$ such that $\nu_{1} \leq \mu_{1}$ and $\nu_{2} \leq \mu_{2}$, where $\nu_{1}, \nu_{2}$ are the marginals of $\nu$. This new supremum in $\nu$ is reached if and only if the original supremum in $\mu$ is reached.

3.6 Lemma. Let $\mu_{1}, \mu_{2}$ be probability measures on $\mathcal{X}_{1}, \mathcal{X}_{2}$ respectively and $B \subset \mathcal{X}_{1} \times \mathcal{X}_{2}$ a Borel set such that

$$
S_{\mu_{1}-\nu_{1}, \mu_{2}-\nu_{2}}(B)=S_{\mu_{1}, \mu_{2}}(B)-\nu(B)
$$

for every positive measure $\nu$ on $B$ such that $\nu_{1} \leq \mu_{1}$ and $\nu_{2} \leq \mu_{2}$, where $\nu_{1}, \nu_{2}$ are the marginals of $\nu$. Then the supremum $S_{\mu_{1}, \mu_{2}}(B)$ is reached.

(See also 8.12.)

Proof. First, taking a positive measure $\nu$ on $B$ such that $\nu_{1} \leq \mu_{1}, \nu_{2} \leq \mu_{2}$ and $\nu(B) \geq$ $\frac{1}{2} S_{\mu_{1}, \mu_{2}}(B)$ we get

$$
S_{\mu_{1}-\nu_{1}, \mu_{2}-\nu_{2}}(B)=S_{\mu_{1}, \mu_{2}}(B)-\nu(B) \leq \frac{1}{2} S_{\mu_{1}, \mu_{2}}(B)
$$

Second, taking a positive measure $\nu^{\prime}$ on $B$ such that $\nu_{1}^{\prime} \leq \mu_{1}-\nu_{1}, \nu_{2}^{\prime} \leq \mu_{2}-\nu_{2}$ and $\nu^{\prime}(B) \geq \frac{1}{2} S_{\mu_{1}-\nu_{1}, \mu_{2}-\nu_{2}}(B)$ we get $\left(\nu+\nu^{\prime}\right)_{1} \leq \mu_{1}$ and $\left(\nu+\nu^{\prime}\right)_{2} \leq \mu_{2}$, thus,

$$
S_{\mu_{1}-\nu_{1}-\nu_{1}^{\prime}, \mu_{2}-\nu_{2}-\nu_{2}^{\prime}}(B)=S_{\mu_{1}, \mu_{2}}(B)-\nu(B)-\nu^{\prime}(B) \leq \frac{1}{4} S_{\mu_{1}, \mu_{2}}(B) .
$$

Continuing this way we get a convergent series of positive measures, $\nu+\nu^{\prime}+\nu^{\prime \prime}+\ldots$; its sum is a measure that reaches the supremum indicated in 3.5(b).

3.7 Lemma. Let a random countable set $\left\{X_{1}, X_{2}, \ldots\right\}$ satisfy

$$
\begin{aligned}
& \text { for every Borel set } B \subset(0,1) \text { of positive measure, } \\
& \qquad B \cap\left\{X_{1}, X_{2}, \ldots\right\} \neq \emptyset \text { a.s. }
\end{aligned}
$$

Then the random set has a selector distributed uniformly on $(0,1)$.

Proof. We apply Theorem 3.4 to $\mathcal{X}_{1}=(0,1)^{\infty}, \mathcal{X}_{2}=(0,1), \mu_{1}$ - the joint distribution of $X_{1}, X_{2}, \ldots, \mu_{2}$ - the uniform distribution on $(0,1)$, and $B$ - the set of all pairs $(x, z)$ satisfying (3.2). By (3.8), $B$ intersects $B_{1} \times B_{2}$ for all Borel sets $B_{1} \subset \mathcal{X}_{1}, B_{2} \subset \mathcal{X}_{2}$ of positive measure. Therefore $I_{\mu_{1}, \mu_{2}}(B)=1$. By the same argument, all absolutely continuous measures $\nu_{1}, \nu_{2}$ on $\mathcal{X}_{1}, \mathcal{X}_{2}$ respectively, such that $\nu_{1}\left(\mathcal{X}_{1}\right)=\nu_{2}\left(\mathcal{X}_{2}\right)$, satisfy $I_{\nu_{1}, \nu_{2}}(B)=\nu_{1}\left(\mathcal{X}_{1}\right)$. By 3.5(a), $S_{\nu_{1}, \nu_{2}}(B)=I_{\nu_{1}, \nu_{2}}(B)$. Thus, the condition of Lemma 3.6 is satisfied (by $\mu_{1}, \mu_{2}$ and $B$ ). By 3.6, some measure $P$ reaches $S_{\mu_{1}, \mu_{2}}(B)=I_{\mu_{1}, \mu_{2}}(B)=1$ and therefore $P$ is the needed selector. 
3.9 Counterexample. In Lemma 3.7 one cannot replace 'a selector' with 'a nonrandomized selector (3.3)'. Randomization is essential!

Let $\Omega=(0,1)$ (with Lebesgue measure) and $\left\{X_{1}(\omega), X_{2}(\omega), \ldots\right\}=\left(\frac{1}{2} \omega+\mathbb{Q}\right) \cap(0,1)$ where $\mathbb{Q} \subset \mathbb{R}$ is the set of rational numbers (and $\frac{1}{2} \omega+\mathbb{Q}$ is its shift by $\frac{1}{2} \omega$ ). Then (3.8) is satisfied (since $B+\mathbb{Q}$ is of full measure), but every selector $Z: \Omega \rightarrow(0,1)$ of the form $Z(\omega)=$ $X_{N(\omega)}(\omega)$ has a nonuniform distribution. Proof: let $A_{q}=\left\{\omega \in(0,1): Z(\omega)-\frac{1}{2} \omega=q\right\}$ for $q \in \mathbb{Q}$, then

$$
\mathbb{P}(Z \in B)=2 \sum_{q \in \mathbb{Q}} \int_{B} \mathbf{1}_{A_{q}}(2(x-q)) \mathrm{d} x,
$$

which shows that the distribution of $Z$ has a density taking on the values $0,2,4, \ldots$ only.

\section{Independence}

According to (2.1), our 'random countable set' $\left\{X_{1}, X_{2}, \ldots\right\}$ can be finite, but cannot be empty. This is why in general we cannot treat the intersection, say, $\left\{X_{1}, X_{2}, \ldots\right\} \cap\left(0, \frac{1}{2}\right)$ as a random countable set.

By a random dense countable subset of $(0,1)$ we mean a random countable subset $\left\{X_{1}, X_{2}, \ldots\right\}$ of $(0,1)$, dense in $(0,1)$ a.s. Equivalently, $\mathbb{P}\left(\exists k a<X_{k}<b\right)=1$ whenever $0 \leq a<b \leq 1$. A random dense countable subset of another interval is defined similarly. It is easy to see that $\left\{X_{1}, X_{2}, \ldots\right\} \cap(a, b)$ is a random dense countable subset of $(a, b)$ whenever $\left\{X_{1}, X_{2}, \ldots\right\}$ is a random dense countable subset of $(0,1)$ and $(a, b) \subset(0,1)$. We call $\left\{X_{1}, X_{2}, \ldots\right\} \cap(a, b)$ a fragment of $\left\{X_{1}, X_{2}, \ldots\right\}$.

It may happen that two (or more) fragments can be described by independent sequences of random variables; such fragments will be called independent. The definition is formulated below in terms of random variables, but could be reformulated in terms of measures on $(0,1)^{\infty}$.

4.1 Definition. Let $\left\{X_{1}, X_{2}, \ldots\right\}$ be a random dense countable subset of $(0,1)$. We say that two fragments $\left\{X_{1}, X_{2}, \ldots\right\} \cap\left(0, \frac{1}{2}\right)$ and $\left\{X_{1}, X_{2}, \ldots\right\} \cap\left[\frac{1}{2}, 1\right)$ of $\left\{X_{1}, X_{2}, \ldots\right\}$ are independent, if there exist random variables $Y_{1}, Y_{2}, \ldots$ (on some probability space) such that

(a) $\left\{Y_{1}, Y_{2}, \ldots\right\}$ is distributed like $\left\{X_{1}, X_{2}, \ldots\right\}$;

(b) $Y_{2 k-1}<\frac{1}{2}$ and $Y_{2 k} \geq \frac{1}{2}$ a.s. for $k=1,2, \ldots$;

(c) the random sequence $\left(Y_{2}, Y_{4}, Y_{6}, \ldots\right)$ is independent of the random sequence $\left(Y_{1}, Y_{3}, Y_{5}, \ldots\right)$.

Similarly we define independence of $n$ fragments $\left\{X_{1}, X_{2}, \ldots\right\} \cap\left[a_{k-1}, a_{k}\right), k=1, \ldots, n$, for any $n=2,3, \ldots$ and any $a_{0}, \ldots, a_{n}$ such that $0=a_{0}<a_{1}<\cdots<a_{n}=1$.

4.2 Definition. A random dense countable subset $\left\{X_{1}, X_{2}, \ldots\right\}$ of $(0,1)$ satisfies the independence condition, if for every $n=2,3, \ldots$ and every $a_{0}, \ldots, a_{n}$ such that $0=$ 
$a_{0}<a_{1}<\cdots<a_{n}=1$ the $n$ fragments $\left\{X_{1}, X_{2}, \ldots\right\} \cap\left[a_{k-1}, a_{k}\right)(k=1, \ldots, n)$ are independent.

Such random dense countable sets are described below, assuming that each $X_{k}$ has a density, in other words,

$$
\begin{aligned}
& \text { for every Borel set } B \subset(0,1) \text { of measure } 0 \text {, } \\
& \qquad B \cap\left\{X_{1}, X_{2}, \ldots\right\}=\emptyset \text { a.s. }
\end{aligned}
$$

(In contrast to Main lemma, existence of joint densities is not assumed. See also 5.10.)

4.4 Proposition. For every random dense countable set satisfying (4.3) and the independence condition there exists a measurable function $r:(0,1) \rightarrow[0, \infty]$ such that for every Borel set $B \subset(0,1)$

(a) if $\int_{B} r(x) \mathrm{d} x=\infty$ then the set $B \cap\left\{X_{1}, X_{2}, \ldots\right\}$ is infinite a.s.;

(b) if $\int_{B} r(x) \mathrm{d} x<\infty$ then the set $B \cap\left\{X_{1}, X_{2}, \ldots\right\}$ is finite a.s., and the number of its elements has the Poisson distribution with the mean $\int_{B} r(x) \mathrm{d} x$.

Note that $r(\cdot)$ may be infinite. See also 9.5 .

The proof is given after some remarks and lemmas.

4.5 Remark. The function $r$ is determined uniquely (up to equality almost everywhere) by the random dense countable set and moreover, by its distribution. That is, if $\left\{Y_{1}, Y_{2}, \ldots\right\}$ is distributed like $\left\{X_{1}, X_{2}, \ldots\right\}$ and $4.4(\mathrm{a}, \mathrm{b})$ hold for $\left\{Y_{1}, Y_{2}, \ldots\right\}$ and another function $r_{1}$, then $r_{1}(x)=r(x)$ for almost all $x \in(0,1)$.

4.6 Remark. The function $r$ is just the sum

$$
r(\cdot)=f_{1}(\cdot)+f_{2}(\cdot)+\ldots
$$

of the densities $f_{k}$ of $X_{k}$.

4.7 Remark. For every measurable function $r:(0,1) \rightarrow[0, \infty]$ such that $\int_{a}^{b} r(x) \mathrm{d} x=$ $\infty$ whenever $0 \leq a<b \leq 1$, there exists a random dense countable set $\left\{X_{1}, X_{2}, \ldots\right\}$ satisfying (4.3), the independence condition and 4.4(a,b) (for the given $r$ ). See also 9.6 and [10, Sect. 2.5].

If $r(x)=\infty$ almost everywhere, we take an unordered infinite sample.

If $r(x)<\infty$ almost everywhere, we take a Poisson point process with the intensity measure $r(x) \mathrm{d} x$.

Otherwise we combine an unordered infinite sample on $\{x: r(x)=\infty\}$ and a Poisson point process on $\{x: r(x)<\infty\}$. 
In order to prove 4.4, for a given $B \subset(0,1)$ we denote by $\xi(x, y)$ the (random) number of elements (maybe, $\infty$ ) in the set $B \cap(x, y) \cap\left\{X_{1}, X_{2}, \ldots\right\}$ and introduce

$$
\begin{aligned}
& \alpha(x, y)=\mathbb{P}(\xi(x, y)=0), \\
& \beta(x, y)=\mathbb{E} \exp (-\xi(x, y))
\end{aligned}
$$

for $0 \leq x<y \leq 1$. (Of course, $\exp (-\infty)=0$.) Clearly,

$$
\left(1-\mathrm{e}^{-1}\right)(1-\alpha(x, y)) \leq 1-\beta(x, y) \leq 1-\alpha(x, y)
$$

for $0 \leq x<y \leq 1$. By (4.3) and the independence condition,

$$
\begin{gathered}
\xi(x, y)+\xi(y, z)=\xi(x, z), \\
\alpha(x, y) \alpha(y, z)=\alpha(x, z), \\
\beta(x, y) \beta(y, z)=\beta(x, z)
\end{gathered}
$$

whenever $0 \leq x<y<z \leq 1$.

4.9 Lemma. If $\beta(0,1) \neq 0$ then $\beta(x-\varepsilon, x+\varepsilon) \rightarrow 1$ as $\varepsilon \rightarrow 0+$ for every $x \in(0,1)$.

Proof. Let $x_{1}<x_{2}<\ldots, x_{k} \rightarrow x$. Random variables $\xi\left(x_{k}, x_{k+1}\right)$ are independent. By Kolmogorov's $0-1$ law, the event $\xi\left(x_{k}, x\right) \rightarrow 0$ is of probability 0 or 1 . It cannot be of probability 0 , since then $\xi\left(x_{1}, x\right)=\infty$ a.s., which implies $\beta(0,1)=0$. Thus, $\xi\left(x_{k}, x\right) \rightarrow 0$ a.s., therefore $\beta\left(x_{k}, x\right) \rightarrow 1$ and $\beta(x-\varepsilon, 1) \rightarrow 1$. Similarly, $\beta(x, x+\varepsilon) \rightarrow 1$.

4.10 Lemma. If $\beta(0,1) \neq 0$ then $\alpha(0,1) \neq 0$.

Proof. By 4.9, for every $x \in(0,1)$ there exists $\varepsilon>0$ such that $\beta(x-\varepsilon, x+\varepsilon)>\mathrm{e}^{-1}$, therefore $\alpha(x-\varepsilon, x+\varepsilon) \neq 0$ by (4.8). (For $x=0, x=1$ we use one-sided neighborhoods.) Choosing a finite covering and using multiplicativity of $\alpha$ we get $\alpha(0,1) \neq 0$.

4.11 Lemma. If $\alpha(0,1) \neq 0$ then $\xi(0,1)$ has the Poisson distribution with the mean $-\ln \alpha(0,1)$.

Proof. By 4.9 and (4.8), $\alpha(x-\varepsilon, x+\varepsilon) \rightarrow 1$. We define a nonatomic finite positive measure $\mu$ on $[0,1]$ by

$$
\mu([x, y])=-\ln \alpha(x, y) \quad \text { for } 0 \leq x<y \leq 1
$$

and introduce a Poisson point process on $[0,1]$ whose intensity measure is $\mu$. Denote by $\eta(x, y)$ the (random) number of Poisson points on $[x, y]$, then $\mathbb{E} \eta(x, y)=\mu([x, y])$ and $\mathbb{P}(\eta(x, y)=0)=\exp (-\mathbb{E} \eta(x, y))=\alpha(x, y)=\mathbb{P}(\xi(x, y)=0)$. In other words, the two random variables $\xi(x, y) \wedge 1$ and $\eta(x, y) \wedge 1$ are identically distributed (of course, $a \wedge b=\min (a, b))$. By independence, for any $n$ the joint distribution of $n$ random variables 
$\xi\left(\frac{k-1}{n}, \frac{k}{n}\right) \wedge 1$ (for $\left.k=1, \ldots, n\right)$ is equal to the joint distribution of $n$ random variables $\eta\left(\frac{k-1}{n}, \frac{k}{n}\right) \wedge 1$. Taking into account that

$$
\xi(0,1)=\lim _{n \rightarrow \infty} \sum_{k=1}^{n}\left(\xi\left(\frac{k-1}{n}, \frac{k}{n}\right) \wedge 1\right)
$$

and the same for $\eta$, we conclude that $\xi(0,1)$ and $\eta(0,1)$ are identically distributed.

4.12 Remark. In addition (but we do not need it),

(a) the joint distribution of $\xi(r, s)$ for all rational $r, s$ such that $0 \leq r<s \leq 1$ (this is a countable family of random variables) is equal to the joint distribution of all $\eta(r, s)$,

(b) the random finite set $B \cap\left\{X_{1}, X_{2}, \ldots\right\}$ is distributed like the Poisson point process,

(c) the measure $\mu$ has the density $\left(f_{1}+f_{2}+\ldots\right) \cdot \mathbf{1}_{B}$, where $f_{k}$ is the density of $X_{k}$.

Proof of Proposition 4.4. We take $r=f_{1}+f_{2}+\ldots$, note that $\int_{B} r(x) \mathrm{d} x=\mathbb{E} \xi(0,1)$ and prove (b) first.

(b) Let $\int_{B} r(x) \mathrm{d} x<\infty$, then $\xi(0,1)<\infty$ a.s., therefore $\beta(0,1) \neq 0$. By $4.10, \alpha(0,1) \neq 0$. By 4.11, $\xi(0,1)$ has a Poisson distribution.

(a) Let $\int_{B} r(x) \mathrm{d} x=\infty$, then $\mathbb{E} \xi(0,1)=\infty$, thus $\xi(0,1)$ cannot have a Poisson distribution. By the argument used in the proof of $(\mathrm{b}), \beta(0,1)=0$. Therefore $\xi(0,1)=\infty$ a.s.

\section{Selectors and independence}

We consider a random dense countable subset $\left\{X_{1}, X_{2}, \ldots\right\}$ of $(0,1)$, satisfying the independence condition and (3.8). If (4.3) is also satisfied then (3.8) means that the corresponding function $r$ (see 4.4) is infinite almost everywhere.

A uniformly distributed selector exists by 3.7. Moreover, there exists a pair of independent uniformly distributed selectors. It follows via Th. 3.4 from the fact that $\left\{X_{1}, X_{2}, \ldots\right\} \times$ $\left\{X_{1}, X_{2}, \ldots\right\}$ intersects a.s. any given set $B \subset(0,1) \times(0,1)$ of positive measure. Hint: we may assume that $B \subset(0, \theta) \times(\theta, 1)$ for some $\theta \in(0,1)$; consider independent fragments $\left\{Y_{1}, Y_{3}, \ldots\right\}=(0, \theta) \cap\left\{X_{1}, X_{2}, \ldots\right\},\left\{Y_{2}, Y_{4}, \ldots\right\}=[\theta, 1) \cap\left\{X_{1}, X_{2}, \ldots\right\}$; almost surely, the first fragment intersects the first projection of $B$, and the second fragment intersects the corresponding section of $B$.

However, we need a stronger statement: for every selector $Z_{1}$ there exists a selector $Z_{2}$ distributed uniformly and independent of $Z_{1}$; here is the exact formulation. (The proof is given after Lemma 5.8.)

5.1 Proposition. Let $\left\{X_{1}, X_{2}, \ldots\right\}$ be a random dense countable subset of $(0,1)$ satisfying the independence condition and (3.8). Let a probability measure $P_{1}$ on $(0,1)^{\infty} \times(0,1)$ be a selector of $\left\{X_{1}, X_{2}, \ldots\right\}$ (as defined by 3.1). Then there exists a probability measure $P_{2}$ 
on $(0,1)^{\infty} \times(0,1)^{2}$ such that, denoting points of $(0,1)^{\infty} \times(0,1)^{2}$ by $\left(x,\left(z_{1}, z_{2}\right)\right)$, we have (w.r.t. $P_{2}$ )

(a) the joint distribution of $x$ and $z_{1}$ is equal to $P_{1}$;

(b) the distribution of $z_{2}$ is uniform on $(0,1)$;

(c) $z_{1}, z_{2}$ are independent;

(d) $z_{2} \in\left\{x_{1}, x_{2}, \ldots\right\}$ a.s. (where $\left(x_{1}, x_{2}, \ldots\right)=x$ ).

Conditioning on $z_{1}$ decomposes the two-selector problem into a continuum of singleselector problems. In terms of conditional distributions $P_{1}\left(\mathrm{~d} x \mid z_{1}\right), P_{2}\left(\mathrm{~d} x \mathrm{~d} z_{2} \mid z_{1}\right)$ we need the following:

(e) $z_{2} \in\left\{x_{1}, x_{2}, \ldots\right\}$ for $P_{2}\left(\cdot \mid z_{1}\right)$-almost all $\left(\left(x_{1}, x_{2}, \ldots\right), z_{2}\right)$;

(f) the distribution of $x$ according to $P_{2}\left(\mathrm{~d} x \mathrm{~d} z_{2} \mid z_{1}\right)$ is $P_{1}\left(\mathrm{~d} x \mid z_{1}\right)$;

(g) the distribution of $z_{2}$ according to $P_{2}\left(\mathrm{~d} x \mathrm{~d} z_{2} \mid z_{1}\right)$ is uniform on $(0,1)$.

That is, we need a uniformly distributed selector of a random set distributed $P_{1}\left(\cdot \mid z_{1}\right)$. To this end we will transfer (3.8) from the unconditional joint distribution of $X_{1}, X_{2}, \ldots$ to their conditional joint distribution $P_{1}\left(\cdot \mid z_{1}\right)$.

5.2 Proposition. Let $X_{1}, X_{2}, \ldots$ and $Y_{1}, Y_{2}, \ldots$ be as in Def. 4.1, and $\mathbb{P}\left(X_{1}<\frac{1}{2}\right)>$ 0 . Then for almost all $x_{1} \in\left(0, \frac{1}{2}\right)$ (w.r.t. the distribution of $\left.X_{1}\right)$, the conditional joint distribution of $Y_{2}, Y_{4}, \ldots$ given $X_{1}=x_{1}$ is absolutely continuous w.r.t. the (unconditional) joint distribution of $Y_{2}, Y_{4}, \ldots$

The proof is given before Lemma 5.7.

5.3 Counterexample. Condition 4.1 (c) is essential for 5.2 (in spite of the fact that $Y_{1}, Y_{3}, \ldots$ are irrelevant).

We take independent random variables $Z_{1}, Z_{2}, \ldots$ such that each $Z_{2 k-1}$ is uniform on $\left(0, \frac{1}{2}\right)$ and each $Z_{2 k}$ is uniform on $\left(\frac{1}{2}, 1\right)$. We define $Y_{1}, Y_{2}, \ldots$ as follows. First, $Y_{2 k-1}=$ $Z_{2 k-1}$. Second, if the $k$-th binary digit of $2 Y_{1}$ is equal to 1 then $Y_{4 k-2}=\min \left(Z_{4 k-2}, Z_{4 k}\right)$ and $Y_{4 k}=\max \left(Z_{4 k-2}, Z_{4 k}\right)$; otherwise (if the digit is 0$), Y_{4 k-2}=\max \left(Z_{4 k-2}, Z_{4 k}\right)$ and $Y_{4 k}=\min \left(Z_{4 k-2}, Z_{4 k}\right)$.

We get a random dense countable set $\left\{Y_{1}, Y_{2}, \ldots\right\}$ whose fragments $\left\{Y_{1}, Y_{2}, \ldots\right\} \cap\left(0, \frac{1}{2}\right)=$ $\left\{Y_{1}, Y_{3}, \ldots\right\}$ and $\left\{Y_{1}, Y_{2}, \ldots\right\} \cap\left(\frac{1}{2}, 1\right)=\left\{Y_{2}, Y_{4}, \ldots\right\}$ are independent. However, $Y_{1}$ is a function of $Y_{2}, Y_{4}, \ldots$ (and of course, the conditional joint distribution of $Y_{2}, Y_{4}, \ldots$ given $Y_{1}$ is singular to their unconditional joint distribution).

In order to prove 5.2 we may partition the event $X_{1}<\frac{1}{2}$ into events $X_{1}=Y_{2 k-1}$. Within such event the condition $X_{1}=x_{1}$ becomes just $Y_{2 k-1}=x_{1}$. However, it does not make the matter trivial, since the event $X_{1}=Y_{2 k-1}$ need not belong to the $\sigma$-field generated by $Y_{1}, Y_{3}, \ldots$ (nor to the $\sigma$-field generated by $X_{1}$ ). 


\section{DIGRESSION: NONSINGULAR PAIRS}

Sometimes dependence between two random variables reduces to a joint density (w.r.t. their marginal distributions). Here are two formulation in general terms.

5.4 Lemma. Let $(\Omega, \mathcal{F}, P)$ be a probability space and $C \subset \Omega$ a measurable set. The following two conditions on a pair of sub- $\sigma$-fields $\mathcal{F}_{1}, \mathcal{F}_{2} \subset \mathcal{F}$ are equivalent:

(a) there exists a measurable function $f: \Omega \times \Omega \rightarrow[0, \infty)$ such that

$$
P(A \cap B \cap C)=\int_{A \times B} f\left(\omega_{1}, \omega_{2}\right) P\left(\mathrm{~d} \omega_{1}\right) P\left(\mathrm{~d} \omega_{2}\right) \quad \text { for all } A \in \mathcal{F}_{1}, B \in \mathcal{F}_{2} ;
$$

(b) there exists a measurable function $g: C \times C \rightarrow[0, \infty)$ such that

$$
P(A \cap B \cap C)=\int_{(A \cap C) \times(B \cap C)} g\left(\omega_{1}, \omega_{2}\right) P\left(\mathrm{~d} \omega_{1}\right) P\left(\mathrm{~d} \omega_{2}\right)
$$

for all $A \in \mathcal{F}_{1}, B \in \mathcal{F}_{2}$.

(Note that $f, g$ may vanish somewhere, and $C$ need not belong to $\mathcal{F}_{1}$ or $\mathcal{F}_{2}$.)

Proof. (b) $\Longrightarrow$ (a): just take $f\left(\omega_{1}, \omega_{2}\right)=g\left(\omega_{1}, \omega_{2}\right)$ for $\omega_{1}, \omega_{2} \in C$ and 0 otherwise.

(a) $\Longrightarrow(\mathrm{b})$ : we consider conditional probabilities $h_{1}=\mathbb{P}\left(C \mid \mathcal{F}_{1}\right), h_{2}=\mathbb{P}\left(C \mid \mathcal{F}_{2}\right)$, note that $h_{1}(\omega)>0, h_{2}(\omega)>0$ for almost all $\omega \in C$ and define

$$
g\left(\omega_{1}, \omega_{2}\right)=\frac{f\left(\omega_{1}, \omega_{2}\right)}{h_{1}\left(\omega_{1}\right) h_{2}\left(\omega_{2}\right)} \quad \text { for } \omega_{1}, \omega_{2} \in C
$$

Then

$$
\begin{aligned}
\int_{(A \cap C) \times(B \cap C)} g\left(\omega_{1}, \omega_{2}\right) P\left(\mathrm{~d} \omega_{1}\right) P\left(\mathrm{~d} \omega_{2}\right) & = \\
& =\int_{A \times B} \frac{f\left(\omega_{1}, \omega_{2}\right) \mathbf{1}_{C}\left(\omega_{1}\right) \mathbf{1}_{C}\left(\omega_{2}\right)}{h_{1}\left(\omega_{1}\right) h_{2}\left(\omega_{2}\right)} P\left(\mathrm{~d} \omega_{1}\right) P\left(\mathrm{~d} \omega_{2}\right) .
\end{aligned}
$$

(The integrand is treated as 0 outside $C \times C$.) Assuming that $f$ is $\left(\mathcal{F}_{1} \otimes \mathcal{F}_{2}\right)$-measurable (otherwise $f$ may be replaced with its conditional expectation) we see that the conditional expectation of the integrand, given $\mathcal{F}_{1} \otimes \mathcal{F}_{2}$, is equal to $f\left(\omega_{1}, \omega_{2}\right)$. Thus, the integral is

$$
\cdots=\int_{A \times B} f\left(\omega_{1}, \omega_{2}\right) P\left(\mathrm{~d} \omega_{1}\right) P\left(\mathrm{~d} \omega_{2}\right)=P(A \cap B \cap C) .
$$

5.5 Definition. Let $(\Omega, \mathcal{F}, P)$ be a probability space and $C \subset \Omega$ a measurable set. Two sub- $\sigma$-fields $\mathcal{F}_{1}, \mathcal{F}_{2} \subset \mathcal{F}$ are a nonsingular pair within $C$, if they satisfy the equivalent conditions of Lemma 5.4. 
5.6 Lemma. (a) Let $C_{1} \subset C_{2}$. If $\mathcal{F}_{1}, \mathcal{F}_{2}$ are a nonsingular pair within $C_{2}$ then they are a nonsingular pair within $C_{1}$.

(b) Let $C_{1}, C_{2}, \ldots$ be pairwise disjoint and $C=C_{1} \cup C_{2} \cup \ldots$ If $\mathcal{F}_{1}, \mathcal{F}_{2}$ are a nonsingular pair within $C_{k}$ for each $k$ then they are a nonsingular pair within $C$.

(c) Let $\mathcal{E}_{1} \subset \mathcal{F}$ be another sub- $\sigma$-field such that $\mathcal{E}_{1} \subset \mathcal{F}_{1}$ within $C$ in the sense that

$$
\forall E \in \mathcal{E}_{1} \quad \exists A \in \mathcal{F}_{1} \quad(A \cap C=E \cap C) .
$$

If $\mathcal{F}_{1}, \mathcal{F}_{2}$ are a nonsingular pair within $C$ then $\mathcal{E}_{1}, \mathcal{F}_{2}$ are a nonsingular pair within $C$.

Proof. (a) We define two measures $\mu_{1}, \mu_{2}$ on $\left(\Omega, \mathcal{F}_{1}\right) \times\left(\Omega, \mathcal{F}_{2}\right)$ by $\mu_{k}(Z)=P\left(C_{k} \cap\{\omega\right.$ : $(\omega, \omega) \in Z\})$ for $k=1$, 2. Clearly, $\mu_{k}(A \times B)=P\left(A \cap B \cap C_{k}\right)$. Condition 5.4(a) for $C_{k}$ means absolute continuity of $\mu_{k}$ (w.r.t. $\left.P\right|_{\mathcal{F}_{1}} \times\left. P\right|_{\mathcal{F}_{2}}$ ). However, $\mu_{1} \leq \mu_{2}$.

(b) Using the first definition, 5.4(a), we just take $f=f_{1}+f_{2}+\ldots$

(c) Immediate, provided that the second definition us used, 5.4(b).

\section{END OF DIGRESSION}

Proof of Prop. 5.2. It is sufficient to prove that the two $\sigma$-fields $\sigma\left(X_{1}\right)$ (generated by $X_{1}$ ) and $\sigma\left(Y_{2}, Y_{4}, \ldots\right)$ are a nonsingular pair within the event $C=\left\{X_{1}<\frac{1}{2}\right\}$. Without loss of generality we assume that $Y_{1}, Y_{3}, \ldots$ are pairwise different a.s. (otherwise we skip redundant elements via a random renumbering). We partition $C$ into events $C_{k}=\left\{X_{1}=\right.$ $\left.Y_{2 k-1}\right\}$. Lemma 5.6(b) reduces $C$ to $C_{k}$. By 5.6(c) we replace $\sigma\left(X_{1}\right)$ with $\sigma\left(Y_{2 k-1}\right)$. By 5.6(a) we replace $C_{k}$ with the whole $\Omega$. Finally, the $\sigma$-fields $\sigma\left(Y_{2 k-1}\right), \sigma\left(Y_{2}, Y_{4}, \ldots\right)$ are a nonsingular pair within $\Omega$, since they are independent.

5.7 Lemma. Let $\left\{X_{1}, X_{2}, \ldots\right\}$ be a random dense countable subset of $(0,1)$ satisfying the independence condition and (3.8). Then for every Borel set $B \subset(0,1)$ of positive measure,

$$
\mathbb{P}\left(\left\{X_{2}, X_{3}, \ldots\right\} \cap B \neq \emptyset \mid X_{1}\right)=1 \quad \text { a.s. }
$$

Proof. First, we assume in addition that mes $\left(B \cap\left(\frac{1}{2}, 1\right)\right)>0$ ('mes' stands for Lebesgue measure) and $\mathbb{P}\left(X_{1}<\frac{1}{2}\right)=1$. Introducing $Y_{k}$ according to Def. 4.1 we note that $\left\{Y_{2}, Y_{4}, \ldots\right\} \cap B \supset\left\{X_{1}, X_{2}, \ldots\right\} \cap B \cap\left(\frac{1}{2}, 1\right) \neq \emptyset$ a.s. by (3.8). It follows via Prop. 5.2 that $\mathbb{P}\left(\left\{Y_{2}, Y_{4}, \ldots\right\} \cap B \neq \emptyset \mid X_{1}\right)=1$ a.s. Taking into account that $\left\{X_{2}, X_{3}, \ldots\right\} \supset$ $\left\{Y_{2}, Y_{4}, \ldots\right\}$ we get $\mathbb{P}\left(\left\{X_{2}, X_{3}, \ldots\right\} \cap B \neq \emptyset \mid X_{1}\right)=1$ a.s.

Similarly we consider the case $\operatorname{mes}\left(B \cap\left(0, \frac{1}{2}\right)\right)>0$ and $\mathbb{P}\left(X_{1} \geq \frac{1}{2}\right)=1$.

Assuming both mes $\left(B \cap\left(0, \frac{1}{2}\right)\right)>0$ and $\operatorname{mes}\left(B \cap\left(\frac{1}{2}, 1\right)\right)>0$ we get the same conclusion for arbitrary distribution of $X_{1}$.

The same arguments work for any threshold $\theta \in(0,1)$ instead of $\frac{1}{2}$. It remains to note that for every $B$ there exists $\theta$ such that both mes $(B \cap(0, \theta))>0$ and $\operatorname{mes}(B \cap(\theta, 1))>0$. 
The claim of Lemma 5.7 is of the form $\forall B\left(\mathbb{P}\left(\ldots \mid X_{1}\right)=1\right.$ a.s. $)$, but the following lemma gives more: $\mathbb{P}\left(\forall B(\ldots) \mid X_{1}\right)=1$ a.s.

5.8 Lemma. Let $\left\{X_{1}, X_{2}, \ldots\right\}$ be a random dense countable subset of $(0,1)$ satisfying the independence condition and (3.8). Denote by $\nu$ the distribution of $X_{1}$ and by $\mu_{x_{1}}$ the conditional joint distribution of $X_{2}, X_{3}, \ldots$ given $X_{1}=x_{1}$. (Of course, $\mu_{x_{1}}$ is welldefined for $\nu$-almost all $x_{1}$.) Then $\nu$-almost all $x_{1} \in(0,1)$ are such that for every Borel set $B \subset(0,1)$ of positive measure,

$$
\mu_{x_{1}}\left(\left\{\left(x_{2}, x_{3}, \ldots\right):\left\{x_{2}, x_{3}, \ldots\right\} \cap B \neq \emptyset\right\}\right)=1 .
$$

Proof. The proof of 5.7 needs only tiny modification, but the last paragraph (about $\theta$ ) needs some attention. The exceptional set of $x_{1}$ may depend on $\theta$, which is not an obstacle since we may use only rational $\theta$. Details are left to the reader.

Proof of Prop. 5.1. We apply Lemma 5.8 to the sequence $\left(Z_{1}, X_{1}, X_{2}, \ldots\right)$ rather than $\left(X_{1}, X_{2}, \ldots\right)$; here $Z_{1}$ is the given selector. More formally, we consider the image of the given measure $P_{1}$ under the map $(0,1)^{\infty} \times(0,1) \rightarrow(0,1)^{\infty}$ defined by $\left(\left(x_{1}, x_{2}, \ldots\right), z_{1}\right) \mapsto$ $\left(z_{1}, x_{1}, x_{2}, \ldots\right)$.

Lemma 5.8 introduces $\nu$ (the distribution of $Z_{1}$ ) and $\mu_{z_{1}}$ (the conditional distribution of $\left(X_{1}, X_{2}, \ldots\right)$ given $\left.Z_{1}=z_{1}\right)$, and states that (3.8) is satisfied by $\mu_{z_{1}}$ for $\nu$-almost all $z_{1}$. Applying 3.7 to $\mu_{z_{1}}$ we get a probability measure $\tilde{\mu}_{z_{1}}$ on $(0,1)^{\infty} \times(0,1)$ such that the first marginal of $\tilde{\mu}_{z_{1}}$ is equal to $\mu_{z_{1}}$, the second marginal of $\tilde{\mu}_{z_{1}}$ is the uniform distribution on $(0,1)$, and $\tilde{\mu}_{z_{1}}$-almost all pairs $\left(\left(x_{1}, x_{2}, \ldots\right), z_{2}\right)$ satisfy $z_{2} \in\left\{x_{1}, x_{2}, \ldots\right\}$.

In order to combine measures $\tilde{\mu}_{z_{1}}$ into a measure $P_{2}$ we need measurability of the map $z_{1} \mapsto \tilde{\mu}_{z_{1}}$.

The set of all probability measures on $(0,1)^{\infty} \times(0,1)$ is a standard Borel space (see [7], Th. (17.24) and the paragraph after it), and the map $\mu \mapsto \mu(B)$ is Borel for every Borel set $B \subset(0,1)^{\infty} \times(0,1)$. (In fact, these maps generate the Borel $\sigma$-field on the space of measures.) It follows easily that the subset $M$ of the space of measures, introduced below, is Borel. Namely, $M$ is the set of all $\mu$ such that the second marginal of $\mu$ is the uniform distribution on $(0,1)$ and $\mu$ is concentrated on the set of $\left(\left(x_{1}, x_{2}, \ldots\right), z_{2}\right)$ such that $z_{2} \in\left\{x_{1}, x_{2}, \ldots\right\}$. Also, the first marginal of $\mu$ is a Borel function of $\mu$ (which means a Borel map from the space of measures on $(0,1)^{\infty} \times(0,1)$ into the similar space of measures on $\left.(0,1)^{\infty}\right)$.

The conditional measure $\mu_{z_{1}}$ is a $\nu$-measurable function of $z_{1}$ defined $\nu$-almost everywhere; it may be chosen to be a Borel map from $(0,1)$ to the space of measures on $(0,1)^{\infty}$. In addition we can ensure that each $\mu_{z_{1}}$ is the first marginal of some $\tilde{\mu}_{z_{1}} \in M$. It follows that these $\tilde{\mu}_{z_{1}} \in M$ can be chosen as a $\nu$-measurable (maybe not Borel, see $[13,5.1 .7]$ ) function of $z_{1}$, by the (Jankov and) von Neumann uniformization theorem, see [7, Sect. 18A] or [13, Sect. 5.5]. 
Now we combine these $\tilde{\mu}_{z_{1}}$ into a probability measure $P_{2}$ on $(0,1)^{\infty} \times(0,1)^{2}$ such that, denoting a point of $(0,1)^{\infty} \times(0,1)^{2}$ by $\left(\left(x_{1}, x_{2}, \ldots\right),\left(z_{1}, z_{2}\right)\right)$ we have: $z_{1}$ is distributed $\nu$, and $P_{2}\left(\mathrm{~d} x \mathrm{~d} z_{2} \mid z_{1}\right)=\tilde{\mu}_{z_{1}}\left(\mathrm{~d} x \mathrm{~d} z_{2}\right)$.

It remains to note that $P_{2}$ satisfies (e), (f), (g) formulated after Prop. 5.1. The first marginal of $\tilde{\mu}_{z_{1}}=P_{2}\left(\cdot \mid z_{1}\right)$ is equal to $\mu_{z_{1}}=P_{1}\left(\cdot \mid z_{1}\right)$, which verifies (f). The second marginal of $\tilde{\mu}_{z_{1}}=P_{2}\left(\cdot \mid z_{1}\right)$ is the uniform distribution on $(0,1)$, which verifies $(\mathrm{g})$. And $z_{2} \in\left\{x_{1}, x_{2}, \ldots\right\}$ almost sure w.r.t. $\tilde{\mu}_{z_{1}}=P_{2}\left(\cdot \mid z_{1}\right)$, which verifies (e).

Prop. 5.1 is a special case $(n=1)$ of Prop. 5.9 below; the latter shows that for every $n$ selectors $Z_{1}, \ldots, Z_{n}$ there exists a selector $Z_{n+1}$ distributed uniformly and independent of $Z_{1}, \ldots, Z_{n}$.

5.9 Proposition. Let $\left\{X_{1}, X_{2}, \ldots\right\}$ be a random dense countable subset of $(0,1)$ satisfying the independence condition and (3.8). Let $n \in\{1,2, \ldots\}$ be given, and $P_{n}$ be a probability measure on $(0,1)^{\infty} \times(0,1)^{n}$ such that

(i) the first marginal of $P_{n}$ is equal to the joint distribution of $X_{1}, X_{2}, \ldots$;

(ii) $P_{n}$-almost all pairs $(x, z), x=\left(x_{1}, x_{2}, \ldots\right), z=\left(z_{1}, \ldots, z_{n}\right)$ are such that $\left\{z_{1}, \ldots, z_{n}\right\} \subset\left\{x_{1}, x_{2}, \ldots\right\}$.

Then there exists a probability measure $P_{n+1}$ on $(0,1)^{\infty} \times(0,1)^{n+1}$ such that, denoting points of $(0,1)^{\infty} \times(0,1)^{n+1}$ by $\left(x,\left(z_{1}, \ldots, z_{n+1}\right)\right)$, we have (w.r.t. $\left.P_{n+1}\right)$

(a) the joint distribution of $x$ and $\left(z_{1}, \ldots, z_{n}\right)$ is equal to $P_{n}$;

(b) the distribution of $z_{n+1}$ is uniform on $(0,1)$;

(c) $z_{n+1}$ is independent of $\left(z_{1}, \ldots, z_{n}\right)$;

(d) $z_{n+1} \in\left\{x_{1}, x_{2}, \ldots\right\}$ a.s. (where $\left.\left(x_{1}, x_{2}, \ldots\right)=x\right)$.

The proof, quite similar to the proof of Prop. 5.1, is left to the reader, but some hints follow. Two independent fragments of a random set are used in 5.8, according to the partition of $(0,1)$ into $(0, \theta)$ and $[\theta, 1)$, where $\theta \in(0,1)$ is rational. One part contains $z_{1}$, the other part contains a portion of the given set $B$ of positive measure. Now, dealing with $z_{1}, \ldots, z_{n}$ we still partition $(0,1)$ in two parts, but they are not just intervals. Rather, each part consists of finitely many intervals with rational endpoints. Still, the independence condition gives us two independent fragments.

Here is another implication of the independence condition. In some sense the proof below is similar to the proof of 5.1, 5.9. There, (3.8) was transferred to conditional distributions via 5.2 . Here we do it with (4.3).

5.10 Lemma. Let $\left\{X_{1}, X_{2}, \ldots\right\}$ be a random dense countable subset of $(0,1)$ satisfying the independence condition and (4.3). If $\mathbb{P}\left(X_{k}=X_{l}\right)=0$ whenever $k \neq l$ then for every $n$ the joint distribution of $X_{1}, \ldots, X_{n}$ is absolutely continuous.

Proof. Once again, I restrict myself to the case $n=2$, leaving the general case to the reader. 
The marginal (one-dimensional) distribution of any $X_{n}$ is absolutely continuous due to (4.3). It is sufficient to prove that the conditional distribution of $X_{2}$ given $X_{1}$ is absolutely continuous, that is, $\mathbb{P}\left(X_{2} \in B \mid X_{1}\right)=0$ a.s. for all negligible $B \subset(0,1)$ simultaneously. By Prop. 5.2 it holds for $X_{1}<\frac{1}{2}$ and $B \subset\left(\frac{1}{2}, 1\right)$. Similarly, it holds for $X_{1}<\theta$ and $B \subset(\theta, 1)$, or $X_{1}>\theta$ and $B \subset(0, \theta)$, for all rational $\theta$ simultaneously. Therefore it holds always.

5.11 Remark. In order to have an absolutely continuous distribution of $X_{1}, \ldots, X_{n}$ for a given $n$, the condition $\mathbb{P}\left(X_{k}=X_{l}\right)=0$ is needed only for $k, l \in\{1, \ldots, n\}, k \neq l$.

\section{Main results}

Recall Definitions 4.2 (the independence condition) and 2.4 (the uniform distribution of a random countable set).

6.1 Theorem. A random dense countable subset $\left\{X_{1}, X_{2}, \ldots\right\}$ of $(0,1)$, satisfying the independence condition, has the uniform distribution if and only if

$$
\mathbb{P}\left(B \cap\left\{X_{1}, X_{2}, \ldots\right\} \neq \emptyset\right)= \begin{cases}0 & \text { if } \operatorname{mes}(B)=0, \\ 1 & \text { if } \operatorname{mes}(B)>0\end{cases}
$$

for all Borel sets $B \subset(0,1)$. (Here 'mes' is Lebesgue measure.)

Proof. If it has the uniform distribution then we may assume that $X_{1}, X_{2}, \ldots$ are independent, uniform on $(0,1)$, which makes (6.2) evident.

Let (6.2) be satisfied. In order to prove that $\left\{X_{1}, X_{2}, \ldots\right\}$ has the uniform distribution, it is sufficient to construct a probability measure $\mu$ on $(0,1)^{\infty} \times(0,1)^{\infty}$ such that the first marginal of $\mu$ is the joint distribution of $X_{1}, X_{2}, \ldots$, the second marginal of $\mu$ satisfies Conditions (a), (b) of Main lemma 1.2 , and $\left\{x_{1}, x_{2}, \ldots\right\}=\left\{z_{1}, z_{2}, \ldots\right\}$ for $\mu$-almost all $\left(\left(x_{1}, x_{2}, \ldots\right),\left(z_{1}, z_{2}, \ldots\right)\right)$.

To this end we construct recursively a consistent sequence of probability measures $\mu_{n}$ on $(0,1)^{\infty} \times(0,1)^{n}$ (with the prescribed first marginal) such that for all $n$,

$$
\left\{x_{1}, \ldots, x_{n}\right\} \subset\left\{z_{1}, \ldots, z_{2 n}\right\} \subset\left\{x_{1}, x_{2}, \ldots\right\}
$$

for $\mu_{2 n}$-almost all $\left(\left(x_{1}, x_{2}, \ldots\right),\left(z_{1}, \ldots, z_{2 n}\right)\right)$, and

$$
z_{2 n+1} \text { is distributed uniformly and independent of } z_{1}, \ldots, z_{2 n}
$$

w.r.t. $\mu_{2 n+1}$, and

$$
z_{1}, \ldots, z_{n} \text { are pairwise different }
$$


$\mu_{n}$-almost everywhere. This is sufficient since, first, (6.3) implies $\left\{x_{1}, x_{2}, \ldots\right\}=$ $\left\{z_{1}, z_{2}, \ldots\right\}$ for $\mu$-almost all $\left(\left(x_{1}, x_{2}, \ldots\right),\left(z_{1}, z_{2}, \ldots\right)\right)$ (here $\mu$ is the measure consistent with all $\mu_{n}$ ); second, $1.2($ a) is ensured by (6.5), Lemma 5.10 and Remark 5.11 (applied to $\left(z_{1}, \ldots, z_{n}, x_{1}, x_{2}, \ldots\right)$ rather than $\left.\left(x_{1}, x_{2}, \ldots\right)\right)$; and third, (6.4) implies $1.2(\mathrm{~b})$.

We choose $\mu_{1}$ by means of Lemma 3.7 .

For constructing $\mu_{2 n}$ we introduce Borel functions $K_{2 n}:(0,1)^{\infty} \times(0,1)^{2 n-1} \rightarrow\{1,2, \ldots\}$, $Z_{2 n}:(0,1)^{\infty} \times(0,1)^{2 n-1} \rightarrow(0,1)$ by

$$
\begin{gathered}
K_{2 n}(x, z)=\min \left\{k: x_{k} \notin\left\{z_{1}, \ldots, z_{2 n-1}\right\}\right\}, \\
Z_{2 n}(x, z)=x_{K_{2 n}(x, z)}
\end{gathered}
$$

of course, $x=\left(x_{1}, x_{2}, \ldots\right)$ and $z=\left(z_{1}, \ldots, z_{2 n-1}\right)$. Less formally, $Z_{2 n}(x, z)$ is the first of $x_{k}$ different from $z_{1}, \ldots, z_{2 n-1}$. We define $\mu_{2 n}$ (consistent with $\mu_{2 n-1}$ ) such that

$$
z_{2 n}=Z_{2 n}\left(\left(x_{1}, x_{2}, \ldots\right),\left(z_{1}, \ldots, z_{2 n-1}\right)\right)
$$

for $\mu_{2 n}$-almost all $(x, z)$. In other words, $\mu_{2 n}$ is the distribution of

$$
\left(\left(x_{1}, x_{2}, \ldots\right),\left(z_{1}, \ldots, z_{2 n-1}, Z_{2 n}(x, z)\right)\right)
$$

where $(x, z)=\left(\left(x_{1}, x_{2}, \ldots\right),\left(z_{1}, \ldots, z_{2 n-1}\right)\right)$ is distributed $\mu_{2 n-1}$. Having $(6.3)$ on the previous step, $\left\{x_{1}, \ldots, x_{n-1}\right\} \subset\left\{z_{1}, \ldots, z_{2 n-2}\right\}$, we conclude that $K_{2 n}(x, z) \geq n$, thus, $\left\{x_{1}, \ldots, x_{n}\right\} \subset\left\{z_{1}, \ldots, z_{2 n-1}, Z_{2 n}(x, z)\right\}$, which ensures (6.3) on the current step.

Finally, we choose $\mu_{2 n+1}$ by means of Prop. 5.9.

6.6 Remark. Assuming (3.8) instead of (6.2) we conclude that some part of $\left\{X_{1}, X_{2}, \ldots\right\}$ (in the sense of 2.5) has the uniform distribution. To this end we use only the 'odd' part of the proof of Th. 6.1, that is, the construction of $\mu_{2 n+1}$.

6.7 Remark. Assuming (4.3) instead of (6.2) we conclude that $\left\{X_{1}, X_{2}, \ldots\right\}$ is distributed as a part of a uniformly distributed random set. To this end we use only the 'even' part of the proof of Th. 6.1, that is, the construction of $\mu_{2 n}$, in combination with Remark 2.5.

6.8 Definition. A random dense countable subset $\left\{X_{1}, X_{2}, \ldots\right\}$ of $(0,1)$, satisfying the independence condition, is stationary, if for every $a, b, c, d \in(0,1)$ such that $b-a=$ $d-c>0$, the two random dense countable sets

$$
\begin{aligned}
& \left\{x \in(0,1): a+(b-a) x \in\left\{X_{1}, X_{2}, \ldots\right\}\right\}, \\
& \left\{x \in(0,1): c+(d-c) x \in\left\{X_{1}, X_{2}, \ldots\right\}\right\}
\end{aligned}
$$

are identically distributed.

6.9 Theorem. Every random dense countable subset of $(0,1)$, satisfying the independence condition and stationary, has the uniform distribution. 
Proof. First we prove (4.3). Let $B \subset(0,1)$ be a Borel set of measure 0. Then for every $x, x \notin B+u$ for almost all $u$. Therefore for every $\omega,\left\{X_{1}(\omega), X_{2}(\omega), \ldots\right\} \cap(B+u)=\emptyset$ for almost all $u$. By Fubini's theorem, $\mathbb{P}\left(\left\{X_{1}, X_{2}, \ldots\right\} \cap(B+u)=\emptyset\right)=1$ for almost all $u$. By stationarity, this probability does not depend on $u$ as long as $B \subset\left(0, \frac{1}{2}\right)$ and $u \in\left[0, \frac{1}{2}\right]$, or $B \subset\left(\frac{1}{2}, 1\right)$ and $u \in\left[-\frac{1}{2}, 0\right] ;(4.3)$ follows.

Second, Prop. 4.4 gives us a function $r:(0,1) \rightarrow[0, \infty]$. By Remark 4.5 and stationarity, this function (or rather, its equivalence class) is shift invariant; thus, $r(x)=\infty$ for almost all $x$, which implies (3.8). It remains to apply Th. 6.1.

It is well-known (see $[5,2.9 .12]$ ) that for almost every Brownian path $w:[0, \infty) \rightarrow \mathbb{R}$ each local minimizer (that is, $x \in(0, \infty)$ such that $w(y) \geq w(x)$ for all $y$ close enough to $x$ ) is a strict local minimizer (it means, $w(y)>w(x)$ for all $y$ close enough to $x$, except for $x$ itself), and all local minimizers are a dense countable set.

6.10 Lemma. There exist Borel functions $X_{1}, X_{2}, \cdots: C[0,1] \rightarrow(0,1)$ such that for almost every Brownian path $w$ (that is, for almost all $w \in C[0,1]$ w.r.t. the Wiener measure) the set $\left\{X_{1}(w), X_{2}(w), \ldots\right\}$ is equal to the set of all local minimizers of $w$.

I give two proofs.

First proof. We take a sequence of intervals $\left(a_{k}, b_{k}\right) \subset(0,1)$ that are a base of the topology, and define $X_{k}(w)$ as the (global) minimizer of $w$ on $\left[a_{k}, b_{k}\right]$ whenever it is unique.

Second proof. It is observed by Kendall [9, Th. 3.4] that well-known selection theorems (see [7, Th. (18.10)]) can be used for constructing $X_{1}, X_{2}, \ldots$ provided that the set of pairs

$$
\{(w, x): x \text { is a local minimizer of } w\}
$$

is a Borel subset of $C[0,1] \times(0,1)$. It remains to note that for every $\varepsilon>0$ the set of pairs $(w, x)$ such that $w(x)=\min _{[x-\varepsilon, x+\varepsilon]} w$ is closed.

6.11 Theorem. The random dense countable set of all local minimizers of a Brownian motion on $(0,1)$ has the uniform distribution.

Proof. We start with Lemma 6.10, note that the independence condition and stationarity hold, and apply Theorem 6.9.

All the arguments can be generalized to higher dimensions and applied to the other, percolation-related, models mentioned in Introduction; see 9.7. 


\section{Borelogy, the new framework}

First of all, two quotations.

It has long been recognized in diverse areas of mathematics that in many important cases such quotient spaces $X / E$ cannot be viewed as reasonable subsets of Polish spaces and therefore the usual methods of topology, geometry, measure theory, etc., are not directly applicable for their study. Thus they are often referred to as singular spaces.

Kechris [6, §2].

In this theory, a differential structure of some set $X$ is defined as the set of all the "differentiable parametrizations" of $X[\ldots]$ The set of these chosen parametrizations is called a diffeology of $X$, and its elements are called the plots of the diffeology. [...] In other words, a diffeology of $X$ says how to "walk" differentiably into $X$. Iglesias-Zemmour [4, beginning of Chapter 1].

I propose borelogy, a solution of the following 'ideological equation',

$$
\frac{\text { borelogical space }}{\text { standard Borel space }}=\frac{\text { diffeological space }}{\text { differentiable manifold }} \text {. }
$$

The set $\operatorname{DCS}(0,1)$ of all dense countable subsets of the interval $(0,1)$ is an example of a singular space in the sense of Kechris (see $F_{2}$ in $[6, \S 8]$ ). It is also an example of a borelogical space, see 7.7 below. By the way, some singular spaces are diffeological spaces, see [4, 1.15].

7.1 Definition. A borelogy on a set $V$ is a set $\mathrm{B}$ of maps $\mathbb{R} \rightarrow V$, such that the following three conditions are satisfied:

(a) for every $b \in \mathrm{B}$,

$$
\text { the set }\{(x, y): b(x)=b(y)\} \text { is a Borel subset of } \mathbb{R}^{2} \text {; }
$$

(b) for every Borel function $f: \mathbb{R} \rightarrow \mathbb{R}$ and every $b \in \mathrm{B}$ their composition $b(f(\cdot))$ belongs to $\mathrm{B}$;

(c) there exists $b \in \mathrm{B}$ such that $b(\mathbb{R})=V$ and the set of compositions $b(f(\cdot))$ (where $f$ runs over all Borel functions $\mathbb{R} \rightarrow \mathbb{R}$ ) is the whole $B$.

7.3 Definition. A borelogical space is a pair $(V, \mathrm{~B})$ of a set $V$ and a borelogy $\mathrm{B}$ on $V$. Elements of $\mathrm{B}$ are called plots of the borelogical space.

7.4 Lemma. Let $b: \mathbb{R} \rightarrow V$ be a surjective map satisfying (7.2), and $\mathrm{B}$ be the set of compositions $b(f(\cdot))$ for all Borel $f: \mathbb{R} \rightarrow \mathbb{R}$. Then $\mathrm{B}$ is a borelogy on $V$. 
Proof. (a): The set $\{(x, y): b(f(x))=b(f(y))\}$ is the inverse image of the Borel set $\{(x, y): b(x)=b(y)\}$ under the Borel map $(x, y) \mapsto(f(x), f(y))$, therefore, a Borel set.

(b): $b(f(g(\cdot)))$ belongs to $\mathrm{B}$, since $f(g(\cdot))$ is a Borel function.

(c): The given $b$ fits by construction.

We see that every surjective map $b: \mathbb{R} \rightarrow V$ satisfying (7.2) generates a borelogy. If $f: \mathbb{R} \rightarrow \mathbb{R}$ is a Borel isomorphism (that is, $f$ is invertible, and $f, f^{-1}$ both are Borel functions) then $b$ and $b(f(\cdot))$ generate the same borelogy.

Recall that an uncountable standard Borel space may be defined as a measurable space, Borel isomorphic to $\mathbb{R}$. Every uncountable standard Borel space $S$ may be used instead of $\mathbb{R}$ in the definition of a borelogy. Such $S$-based borelogies are in a natural one-toone correspondence with $\mathbb{R}$-based borelogies. The correspondence is established via an isomorphism between $S$ and $\mathbb{R}$, but does not depend on the choice of the isomorphism.

According to 7.1(c), every borelogy B contains a generating plot, that is, B is generated by some $b: \mathbb{R} \rightarrow V$. Such $b$ establishes a bijective correspondence between $V$ and the quotient set $\mathbb{R} / E_{b}$, where $E_{b}=\{(x, y): b(x)=b(y)\} \subset \mathbb{R}^{2}$ is the relevant equivalence relation (a Borel equivalence relation, due to (7.2)). Every $b^{\prime} \in \mathrm{B}$ is of the form $b^{\prime}(\cdot)=b(f(\cdot))$ for some Borel $f: \mathbb{R} \rightarrow \mathbb{R}$. Clearly, $(x, y) \in E_{b^{\prime}}$ if and only if $(f(x), f(y)) \in E_{b}$. In terms of $[6, \S 3]$ it means that $f$ is a Borel reduction of $E_{b^{\prime}}$ into $E_{b}$, and $\mathbb{R} / E_{b^{\prime}}$ has Borel cardinality at most that of $\mathbb{R} / E_{b}$. It may happen that also $b^{\prime}$ generates $B$. Then one says that $\mathbb{R} / E_{b^{\prime}}$ and $\mathbb{R} / E_{b}$ have the same Borel cardinality [6, §3]. We see that every borelogical space has its (well-defined) Borel cardinality.

Recall also that a standard Borel space is either an uncountable standard Borel space (discussed above), or a finite or countable set equipped with the $\sigma$-field of all subsets. Let $(V, \mathcal{B})$ be a standard Borel space (here $\mathcal{B}$ is a given $\sigma$-field of subsets of $V$ ). We turn it into a borelogical space $(V, \mathrm{~B})$ where $\mathrm{B}$ consists of all Borel maps $b: \mathbb{R} \rightarrow V$. Such a borelogical space will be called nonsingular. This way, standard Borel spaces may be treated as a special case of borelogical spaces. In terms of the equivalence relation $E_{b}$ corresponding to a generating plot $b$, the borelogical space is nonsingular if and only if $E_{b}$ is smooth (or tame), see $[6, \S 6]$. Otherwise, the borelogical space will be called singular.

A nonempty finite or countable set $V$ carries one and only one borelogy $\mathrm{B}$, and $(V, \mathrm{~B})$ is nonsingular.

Pinciroli [12, Def. 1.2] defines a quotient Borel space as a couple $(S, E)$ where $S$ is a standard Borel space and $E$ is a Borel equivalence relation on $S$ whose equivalence classes are countable. He stipulates that the underlying set of $(S, E)$ is the quotient set $S / E$. Clearly, every quotient Borel space is a borelogical space. On the other hand, every borelogical space is of the form $S / E$, however, a freedom is left in the choice of $S$ and $E$ (see also Example 7.8), and $E$ need not have countable equivalence classes.

7.5 Example. The set $V=\mathbb{R} / \mathbb{Q}$ (reals modulo rationals) consists of equivalence classes $\mathbb{Q}+x=\{q+x: q \in \mathbb{Q}\}$ for all $x \in \mathbb{R}$. The natural map $\mathbb{R} \rightarrow V, x \mapsto \mathbb{Q}+x$, generates a borelogy B on $V$ (by Lemma 7.4), and $(V, \mathrm{~B})$ is singular (see Remark 8.9). 
7.6 Example. The set $V=\operatorname{CS}(0,1)$ of all countable subsets of the interval $(0,1)$ is the image of the set $S=(0,1)_{\neq}^{\infty} \subset(0,1)^{\infty}$ of all sequences $\left(s_{1}, s_{2}, \ldots\right)$ of pairwise different numbers $s_{k} \in(0,1)$ under the natural map $b: S \rightarrow V, b\left(s_{1}, s_{2}, \ldots\right)=\left\{s_{1}, s_{2}, \ldots\right\}$.

The set $S$ is a Borel subset of $(0,1)^{\infty}$ (since $\left\{\left(s_{1}, s_{2}, \ldots\right): s_{k} \neq s_{l}\right\}$ is open whenever $k \neq l)$, therefore, a standard Borel space, see [7, Sect. 12.B].

The map $b: S \rightarrow V$ satisfies (7.2), that is, the set of all pairs $\left(s, s^{\prime}\right)=$ $\left(\left(s_{1}, s_{2}, \ldots\right),\left(s_{1}^{\prime}, s_{2}^{\prime}, \ldots\right)\right)$ such that $b(s)=b\left(s^{\prime}\right)$ is a Borel subset of $S \times S$, since

$$
b(s)=b\left(s^{\prime}\right) \Longleftrightarrow \forall k \exists l\left(s_{k}=s_{l}^{\prime}\right) \& \forall k \exists l\left(s_{k}^{\prime}=s_{l}\right) .
$$

By an evident generalization of Lemma 7.4, $b$ generates on $V$ an $S$-based borelogy, which turns $V$ into a borelogical space. It is singular (see Remark 8.9).

7.7 Example. The borelogical space $\operatorname{DCS}(0,1)$ of all dense countable subsets of $(0,1)$ is defined similarly. It is singular (see Remark 8.9).

7.8 Example. The borelogical space $\operatorname{FCS}(0,1)$ of all finite or countable subsets of $(0,1)$ is defined similarly. We may adopt finite sets by replacing $(0,1)_{\neq}^{\infty}$ with $\cup_{n=0,1,2, \ldots ; \infty}(0,1)_{\neq}^{n}$, where $(0,1)_{\neq}^{0}$ contains a single element (the empty sequence) whose image in $\operatorname{FCS}(0,1)$ is the empty set. Alternatively we may adopt finite sets by replacing $(0,1)_{\neq}^{\infty}$ with $(0,1)^{\infty}$, thus permitting equal numbers in the sequences; however, in this case we should bother about the empty set as an element of $\operatorname{FCS}(0,1)$.

We may also consider the set $S$ of all discrete finite positive Borel measures on $(0,1)$ ('discrete' means existence of a countable set of full measure) together with the equivalence relation $E$ of mutual absolute continuity. Once again, $S / E=\mathrm{FCS}(0,1)$. Sketch of the proof. On one hand, a sequence $\left(s_{1}, s_{2}, \ldots\right)$ leads to a discrete measure $A \mapsto \sum_{k: s_{k} \in A} 2^{-k}$. On the other hand, a discrete measure $\mu$ leads to the sequence of its atoms, the most massive atom being the first and so on. (If several atoms are equally massive, the leftmost one is the first.)

As usual, we often say 'a borelogical space $V$ ' rather than 'a borelogical space $(V, \mathrm{~B})$ '.

7.9 Definition. Let $V, W$ be borelogical spaces.

(a) A morphism of $V$ to $W$ is a map $f: V \rightarrow W$ such that for every plot $b$ of $V$ the map $f(b(\cdot))$ is a plot of $W$.

(b) An isomorphism between $V$ and $W$ is an invertible map $f: V \rightarrow W$ such that $f$ and $f^{-1}$ are morphisms.

Choosing generating plots $b$ for $V$ and $b^{\prime}$ for $W$ we observe that $f: V \rightarrow W$ is a morphism if and only if $f(b(\cdot))=b^{\prime}(g(\cdot))$ for some Borel $g: \mathbb{R} \rightarrow \mathbb{R}$.

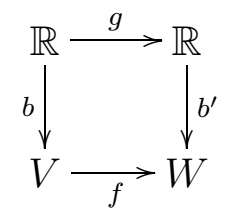


(Compare it with a Borel morphism of Borel equivalence relations [12, p. 1].) If a morphism $f: V \rightarrow W$ is injective (that is, $x_{1} \neq x_{2}$ implies $f\left(x_{1}\right) \neq f\left(x_{2}\right)$ ) then

$$
\left(x_{1}, x_{2}\right) \in E_{b} \Longleftrightarrow\left(g\left(x_{1}\right), g\left(x_{2}\right)\right) \in E_{b^{\prime}},
$$

which means that $g$ is a Borel reduction of $E_{b}$ into $E_{b^{\prime}}$, and the Borel cardinality of $V$ is at most that of $W$. It follows that isomorphic borelogical spaces have the same Borel cardinality. (Is the converse true? I do not know.) Existence of a continuum of (different) Borel cardinalities, mentioned in [6, §6], implies existence of a continuum of mutually nonisomorphic borelogical spaces. Of course, they are singular; all nonsingular borelogical spaces of the same cardinality (finite, countable or continuum) are isomorphic. The Borel cardinality of $\mathbb{R} / \mathbb{Q}$ is well-known as $E_{0}[6, \S 3]$. The Borel cardinality of $\operatorname{CS}(0,1)$ is well-known as $F_{2}[6, \S 8]$.

7.10 Definition. The product of two borelogical spaces $\left(V_{1}, \mathrm{~B}_{1}\right),\left(V_{2}, \mathrm{~B}_{2}\right)$ is the borelogical space $\left(V_{1} \times V_{2}, \mathrm{~B}_{1} \times \mathrm{B}_{2}\right)$ where $\mathrm{B}_{1} \times \mathrm{B}_{2}$ consists of all maps $\mathbb{R} \rightarrow V_{1} \times V_{2}$ of the form $b_{1} \times b_{2}$, that is, $x \mapsto\left(b_{1}(x), b_{2}(x)\right)$, where $b_{1} \in \mathrm{B}_{1}, b_{2} \in \mathrm{B}_{2}$.

7.11 Lemma. Definition 7.10 is correct, that is, $\mathrm{B}_{1} \times \mathrm{B}_{2}$ is a borelogy on $V_{1} \times V_{2}$.

Proof. We check the three conditions of 7.1 .

(a) the set $\left\{(x, y):\left(b_{1}(x), b_{2}(x)\right)=\left(b_{1}(y), b_{2}(y)\right)\right\}=\left\{(x, y): b_{1}(x)=b_{1}(y)\right\} \cap\{(x, y)$ : $\left.b_{2}(x)=b_{2}(y)\right\}$ is the intersection of two Borel sets;

(b) $\left(b_{1} \times b_{2}\right)(f(\cdot))=\left(b_{1}(f(\cdot)), b_{2}(f(\cdot))\right)=b_{1}(f(\cdot)) \times b_{2}(f(\cdot)) \in \mathrm{B}_{1} \times \mathrm{B}_{2}$;

(c) choosing generating plots $b_{1}: \mathbb{R} \rightarrow V_{1}, b_{2}: \mathbb{R} \rightarrow V_{2}$ and a Borel isomorphism $g$ : $\mathbb{R} \rightarrow \mathbb{R}^{2}, g(\cdot)=\left(g_{1}(\cdot), g_{2}(\cdot)\right)$, we define $b: \mathbb{R} \rightarrow V_{1} \times V_{2}$ by $b(x)=\left(b_{1}\left(g_{1}(x)\right), b_{2}\left(g_{2}(x)\right)\right)$ and note that every element of $\mathrm{B}_{1} \times \mathrm{B}_{2}$ is of the form $x \mapsto\left(b_{1}\left(f_{1}(x)\right), b_{2}\left(f_{2}(x)\right)\right)=$ $\left(b_{1}\left(g_{1}(f(x))\right), b_{2}\left(g_{2}(f(x))\right)\right)=b(f(x))$ where $f(x)=g^{-1}\left(\left(f_{1}(x), f_{2}(x)\right)\right)$.

7.12 Remark. Having usual ( $\mathbb{R}$-based) generating plots $b_{1}: \mathbb{R} \rightarrow V_{1}, b_{2}: \mathbb{R} \rightarrow V_{2}$ we get immediately an $\mathbb{R}^{2}$-based generating plot $b: \mathbb{R}^{2} \rightarrow V_{1} \times V_{2}, b(x, y)=\left(b_{1}(x), b_{2}(y)\right)$. However, in order to get a generating plot $\mathbb{R} \rightarrow V_{1} \times V_{2}$ we need a Borel isomorphism between $\mathbb{R}$ and $\mathbb{R}^{2}$.

More generally, having generating plots $b_{1}: S_{1} \rightarrow V_{1}, b_{2}: S_{2} \rightarrow V_{2}$ we get immediately a generating plot $b: S_{1} \times S_{2} \rightarrow V_{1} \times V_{2}, b(x, y)=\left(b_{1}(x), b_{2}(y)\right)$; here $S_{1}, S_{2}$ are standard Borel spaces.

Compare Def. 7.10 with [12, Def. 1.3].

7.13 Example. Defining a borelogical space $\mathbb{R}^{2} / \mathbb{Q}^{2}$ similarly to 7.5 we get (up to a natural isomorphism)

$$
(\mathbb{R} / \mathbb{Q}) \times(\mathbb{R} / \mathbb{Q})=\mathbb{R}^{2} / \mathbb{Q}^{2}
$$

7.14 Example. Defining a borelogical space $\operatorname{CS}[a, b)$ for any $[a, b) \subset \mathbb{R}$ similarly to 7.6 we get (up to a natural isomorphism)

$$
\mathrm{CS}[a, b) \times \mathrm{CS}[b, c)=\mathrm{CS}[a, c)
$$

and the same for DCS and FCS. 


\section{Probability measures on singular spaces}

Each borelogical space $V$ carries a $\sigma$-field $\Sigma$ consisting of all $A \subset V$ such that $b^{-1}(A)$ is a Borel subset of $\mathbb{R}$ for every plot $b: \mathbb{R} \rightarrow V$. Choosing a generating plot $b$ we see that $A \in \Sigma$ if and only if $b^{-1}(A)$ is a Borel set. (That is, $\Sigma$ is the quotient $\sigma$-field [13, Sect. 5.1], see also [12, Sect. 1].) If $V$ is nonsingular then $\Sigma$ is its given Borel $\sigma$-field (since the identical map $V \rightarrow V$ is a plot), thus, $(V, \Sigma)$ is a standard Borel space. If $V$ is singular then it may happen that $(V, \Sigma)$ is still a standard Borel space (which can be shown by means of a well-known counterexample $[13,5.1 .7])$, but in such cases as $\mathbb{R} / \mathbb{Q}$ and $\operatorname{DCS}(0,1)$ it is not.

In my opinion, a notion defined via a singular space can be useful in probability theory only if it admits an equivalent definition in terms of standard spaces. A quote from Pinciroli [12, p. 2]: "[...] the 'right' notion of Borelness for [...] functions between quotient Borel spaces is not the usual one from the context of measurable spaces and maps: here again we want to exploit the original standard Borel structures."

Three examples follow.

First, it may be tempting to define a random element of a borelogical space $V$ as a measurable map from a standard probability space $\Omega$ to the (nonstandard) measurable space $(V, \Sigma)$. However, I prefer to define a random element of $V$ as a map $\Omega \rightarrow V$ of the form $b(X(\cdot))$ where $X: \Omega \rightarrow \mathbb{R}$ is a (usual) random variable, and $b: \mathbb{R} \rightarrow V$ is a plot. Are these two definitions equivalent? I do not know. Every $b(X(\cdot))$ is $\Sigma$-measurable, but I doubt that every $\Sigma$-measurable map is of the form $b(X(\cdot))$. For Borel maps the answer is negative, but for equivalence classes it may be different.

Second, it may be tempting to define the distribution of a random element $b(X(\cdot))$ as the corresponding probability measure on the (nonstandard) measurable space $(V, \Sigma)$. Then two random elements may be treated as identically distributed if their distributions are equal. An equivalent definition in terms of standard spaces will be given (Th. 8.2, Def. 8.3).

Third, it may be tempting to say that two random elements $b(X)$ and $b(Y)$ are independent if $\mathbb{P}(b(X) \in A, b(Y) \in B)=\mathbb{P}(b(X) \in A) \mathbb{P}(b(Y) \in B)$ for all $A, B \in \Sigma$. However, I prefer a different, nonequivalent definition (see Def. 8.13 and Counterexample 8.14).

Recall that a standard probability space (known also as a Lebesgue-Rokhlin space) is a probability space isomorphic (mod 0) to an interval with the Lebesgue measure, a finite or countable collection of atoms, or a combination of both. Every probability measure on a standard Borel space turns it (after completion, that is, adding all negligible sets to the $\sigma$-field) into a standard probability space, see [7, Sect. 17.F] or [13, Th. 3.4.23].

8.1 Definition. Let $V$ be a borelogical space and $\Omega$ a standard probability space. A $V$-valued random variable on $\Omega$ (called also a random element of $V$ ) is an equivalence class of maps $X: \Omega \rightarrow V$ representable in the form $X(\cdot)=b(Y(\cdot))$ for some plot $b: \mathbb{R} \rightarrow V$ and some (usual) random variable $Y: \Omega \rightarrow \mathbb{R}$. (Equivalence means equality almost everywhere on $\Omega$.) 
Choosing a generating plot $b: \mathbb{R} \rightarrow V$ we observe that every random element of $V$ is of the form $b(Y(\cdot))$ with the chosen $b$ and arbitrary $Y$, since $b(f(Y(\cdot)))$ is of this form.

8.2 Theorem. Let $\left(\Omega_{1}, \mathcal{F}_{1}, P_{1}\right),\left(\Omega_{2}, \mathcal{F}_{2}, P_{2}\right)$ be standard probability spaces, $V$ a borelogical space, and $X_{1}, X_{2}$ be $V$-valued random variables on $\Omega_{1}, \Omega_{2}$ respectively. Then the following two conditions are equivalent.

(a) $\mathbb{P}\left(X_{1} \in A\right)=\mathbb{P}\left(X_{2} \in A\right)$ for all $A \in \Sigma$;

(b) there exists a probability measure $P$ on $\Omega_{1} \times \Omega_{2}$ whose marginals are $P_{1}, P_{2}$, such that $X_{1}\left(\omega_{1}\right)=X_{2}\left(\omega_{2}\right)$ for P-almost all $\left(\omega_{1}, \omega_{2}\right)$.

The proof is given after 8.12 .

Theorem 8.2 shows that items (b1), (b2) of the following definition are basically the same.

8.3 Definition. Let $V$ be a borelogical space.

(a) Two $V$-valued random variables are identically distributed, if they satisfy the equivalent conditions (a), (b) of Theorem 8.2.

(b1) A distribution on $V$ is an equivalence class of $V$-valued random variables on the probability space $\Omega=(0,1)$ (with Lebesgue measure); here random variables are treated as equivalent if they are identically distributed.

(b2) A distribution on $V$ is a probability measure on the (generally, nonstandard) measurable space $(V, \Sigma)$, representable in the form $\mathbb{P}(X \in \cdot)$ for some $V$-valued random variable $X$.

(c) A distribution on $V$ is called an $0-1$ distribution, if it ascribes to all sets of $\Sigma$ the probabilities 0,1 only.

8.4 Remark. If $X(\cdot)=b(Y(\cdot))$ has a $0-1$ distribution and $Y^{\prime}$ has a distribution absolutely continuous w.r.t. the distribution of $Y$, then $X^{\prime}=b\left(Y^{\prime}\right)$ and $X$ are identically distributed.

8.5 Example. Continuing Example 7.5 we consider the borelogical space $\mathbb{R} / \mathbb{Q}$ and its generating plot $b: \mathbb{R} \rightarrow \mathbb{R} / \mathbb{Q}, b(x)=\mathbb{Q}+x$. Every random variable $Y: \Omega \rightarrow \mathbb{R}$ leads to a $\mathbb{R} / \mathbb{Q}$-valued random variable $X=\mathbb{Q}+Y$. If the distribution of $Y$ is absolutely continuous then $X$ has a $0-1$ distribution (since for every $\mathbb{Q}$-invariant Borel $B \subset \mathbb{R}$ it is well-known that either $B$ or $\mathbb{R} \backslash B$ is of Lebesgue measure 0 ). All absolutely continuous distributions on $\mathbb{R}$ correspond to a single distribution on $\mathbb{R} / \mathbb{Q}$. This special $0-1$ distribution on $\mathbb{R} / \mathbb{Q}$ may be called the uniform distribution on $\mathbb{R} / \mathbb{Q}$.

8.6 Corollary. For every two absolutely continuous probability measures $\mu, \nu$ on $\mathbb{R}$ there exist random variables $X, Y$ distributed $\mu, \nu$ respectively and such that the difference $X-Y$ is a.s. a (random) rational number.

If $X_{1}(\cdot)=b\left(Y_{1}(\cdot)\right), X_{2}(\cdot)=b\left(Y_{2}(\cdot)\right)$ and $Y_{1}, Y_{2}$ are identically distributed then, of course, $X_{1}, X_{2}$ are identically distributed. The converse does not hold (without an appropriate enlargement of probability spaces), see below. 
8.7 Counterexample. There exist two identically distributed $\mathbb{R} / \mathbb{Q}$-valued random variables $X_{1}, X_{2}: \Omega \rightarrow \mathbb{R} / \mathbb{Q}$ that are not of the form $X_{1}=\mathbb{Q}+Y_{1}, X_{2}=\mathbb{Q}+Y_{2}$ where $Y_{1}, Y_{2}: \Omega \rightarrow \mathbb{R}$ are identically distributed.

Proof. (See also 3.9.) We take $\Omega=(0,1)$ with Lebesgue measure and define for $\omega \in \Omega$

$$
X_{1}(\omega)=\mathbb{Q}+\omega, \quad X_{2}(\omega)=\mathbb{Q}+\sqrt{2} \omega .
$$

Let $Y_{1}: \Omega \rightarrow \mathbb{R}$ satisfy $X_{1}=\mathbb{Q}+Y_{1}$ a.s.; I claim that the distribution of $Y_{1}$ necessarily has an integer-valued density. Similarly, I claim that every $Y_{2}: \Omega \rightarrow \mathbb{R}$ satisfying $X_{2}=$ $\mathbb{Q}+Y_{2}$ a.s. necessarily has a density that takes on the values $0,1 / \sqrt{2}, 2 / \sqrt{2}, 3 / \sqrt{2}, \ldots$ only. Clearly, such $Y_{1}, Y_{2}$ cannot be identically distributed; it remain to prove the first claim (the second claim is similar).

We have $\mathbb{Q}+\omega=\mathbb{Q}+Y_{1}(\omega)$, that is, $Y_{1}(\omega)-\omega \in \mathbb{Q}$. We partition $(0,1)$ into countably many measurable sets $A_{q}=\left\{\omega: Y_{1}(\omega)-\omega=q\right\}$ for $q \in \mathbb{Q}$ and observe that $\mathbb{P}\left(Y_{1} \in B\right)=$ $\sum_{q \in \mathbb{Q}} \operatorname{mes}\left\{\omega \in A_{q}: \omega+q \in B\right\}=\int_{B} f(x) \mathrm{d} x$ where $f(x)$ is the number of $q \in \mathbb{Q}$ such that $x-q \in A_{q}$.

8.8 Example. Continuing Example 7.6 we consider the borelogical space $\operatorname{CS}(0,1)$ and its generating plot $b:(0,1) \underset{\neq}{\infty} \rightarrow \operatorname{CS}(0,1)$. An $(0,1)_{\neq}^{\infty}$-valued random variable $Y$ is nothing but a sequence of random variables $Y_{1}, Y_{2}, \cdots: \Omega \rightarrow(0,1)$ such that $\mathbb{P}\left(Y_{k}=Y_{l}\right)=0$ for $k \neq l$. Every such $Y$ leads to a $\operatorname{CS}(0,1)$-valued random variable $X=b(Y)$, that is, a random countable set $X(\omega)=\left\{Y_{1}(\omega), Y_{2}(\omega), \ldots\right\}$; see also (2.1). If $Y_{k}$ are independent then $X$ has a $0-1$ distribution by the Hewitt-Savage $0-1$ law. Using 8.4, similarly to 8.6, if $Y_{k}$ are independent and the distribution of $Y^{\prime}=\left(Y_{1}^{\prime}, Y_{2}^{\prime}, \ldots\right)$ is absolutely continuous w.r.t. the distribution of $Y=\left(Y_{1}, Y_{2}, \ldots\right)$, then there exists a joining between $Y$ and $Y^{\prime}$ such that $\left\{Y_{1}(\omega), Y_{2}(\omega), \ldots\right\}=\left\{Y_{1}^{\prime}(\omega), Y_{2}^{\prime}(\omega), \ldots\right\}$ a.s.

According to Main lemma 1.2 , a wide class of probability distributions on $(0,1)_{\neq}^{\infty}$ (many of them being mutually singular) corresponds to a single $0-1$ distribution on $\operatorname{CS}(0,1)$, called uniform according to 2.4. (See also 8.5.)

8.9 Remark. Every 0-1 distribution on a standard Borel space (that is, a nonsingular borelogical space) is concentrated at a single point. Therefore, existence of a $0-1$ distribution that does not charge points implies singularity of a borelogical space. (See also $[12$, Remark 3.3].) In particular, $\mathbb{R} / \mathbb{Q}$ and $\operatorname{DCS}(0,1)$ are singular (recall 8.5 and 8.8).

The proof of Theorem 8.2 is based on Kellerer's Theorem 3.4; recall it: $S_{\mu_{1}, \mu_{2}}(B)=$ $I_{\mu_{1}, \mu_{2}}(B)$. The theorem holds for all standard Borel spaces $\mathcal{X}_{1}, \mathcal{X}_{2}$ and Borel sets $B \subset$ $\mathcal{X}_{1} \times \mathcal{X}_{2}$; we apply it to $\mathcal{X}_{1}=\mathcal{X}_{2}=\mathbb{R}$ and specialize the Borel set as follows.

Let a Borel set $E \subset \mathbb{R}^{2}$ be (the graph of) an equivalence relation on $\mathbb{R}$; we introduce the $\sigma$-field $\mathcal{E}$ of all Borel sets $A \subset \mathbb{R}$ that are saturated (invariant) in the sense that

$$
(x, y) \in E \quad \Longrightarrow \quad(x \in A \Longleftrightarrow y \in A) \quad \text { for } x, y \in \mathbb{R} .
$$


8.10 Lemma. For all probability measures $\mu_{1}, \mu_{2}$ on $\mathbb{R}$,

$$
I_{\mu_{1}, \mu_{2}}(E)=1-\sup _{A \in \mathcal{E}}\left|\mu_{1}(A)-\mu_{2}(A)\right| .
$$

Proof. First, " $\leq$ ": we have $E \subset(A \times \mathbb{R}) \cup(\mathbb{R} \times \bar{A})$ for $A \in \mathcal{E}$ (here $\bar{A}=\mathbb{R} \backslash A$ ), therefore $I_{\mu_{1}, \mu_{2}}(E) \leq \mu_{1}(A)+\mu_{2}(\bar{A})=1-\left(\mu_{2}(A)-\mu_{1}(A)\right)$. Similarly, $I_{\mu_{1}, \mu_{2}}(E) \leq$ $1-\left(\mu_{1}(A)-\mu_{2}(A)\right)$.

Second, " $\geq$ ". Let Borel $B_{1}, B_{2}$ satisfy $E \subset\left(B_{1} \times \mathbb{R}\right) \cup\left(\mathbb{R} \times B_{2}\right)$, then $\bar{B}_{1} \times \bar{B}_{2}$ does not intersect $E$. It follows (see [13, Th. 4.4.5] or [7, Exercise (14.14)]) that there exists $A \in \mathcal{E}$ such that $\bar{B}_{1} \subset A, \bar{B}_{2} \subset \bar{A}$. We have $\mu_{1}\left(B_{1}\right)+\mu_{2}\left(B_{2}\right) \geq \mu_{1}(\bar{A})+\mu_{2}(A)=$ $1-\left(\mu_{1}(A)-\mu_{2}(A)\right) \geq 1-\sup _{A \in \mathcal{E}}\left|\mu_{1}(A)-\mu_{2}(A)\right|$.

8.11 Remark. If $\mu_{1}, \mu_{2}$ are finite positive (not just probability) measures such that $\mu_{1}(\mathbb{R})=\mu_{2}(\mathbb{R})$ then $I_{\mu_{1}, \mu_{2}}(E)=\mu_{1}(\mathbb{R})-\sup _{A \in \mathcal{E}}\left|\mu_{1}(A)-\mu_{2}(A)\right|$.

8.12 Lemma. The supremum $S_{\mu_{1}, \mu_{2}}(E)$ is reached for all probability measures $\mu_{1}, \mu_{2}$ on $\mathbb{R}$.

Proof. We check the condition of Lemma 3.6. Let $\nu$ be a positive measure on $E$ with marginals $\nu_{1} \leq \mu_{1}, \nu_{2} \leq \mu_{2}$. By Theorem 3.4 it is sufficient to prove that $I_{\mu_{1}-\nu_{1}, \mu_{2}-\nu_{2}}(E)=$ $I_{\mu_{1}, \mu_{2}}(E)-\nu(E)$. By 8.10 and 8.11 it boils down to the equality

$$
\sup _{A \in \mathcal{E}}\left|\left(\mu_{1}-\nu_{1}\right)(A)-\left(\mu_{2}-\nu_{2}\right)(A)\right|=\sup _{A \in \mathcal{E}}\left|\mu_{1}(A)-\mu_{2}(A)\right| .
$$

It remains to note that $\nu_{1}(A)=\nu_{2}(A)$ for all $A \in \mathcal{E}$.

Proof of Theorem 8.2. (b) $\Longrightarrow$ (a): $P_{1}\left(\left\{\omega_{1}: X_{1}\left(\omega_{1}\right) \in A\right\}\right)=P\left(\left\{\left(\omega_{1}, \omega_{2}\right): X_{1}\left(\omega_{1}\right) \in\right.\right.$ $A\})=P\left(\left\{\left(\omega_{1}, \omega_{2}\right): X_{2}\left(\omega_{2}\right) \in A\right\}\right)=P_{2}\left(\left\{\omega_{2}: X_{2}\left(\omega_{2}\right) \in A\right\}\right)$.

(a) $\Longrightarrow$ (b): We choose a generating plot $b: \mathbb{R} \rightarrow V$ and random variables $Y_{1}: \Omega_{1} \rightarrow \mathbb{R}$, $Y_{2}: \Omega_{2} \rightarrow \mathbb{R}$ such that $X_{1}(\cdot)=b\left(Y_{1}(\cdot)\right), X_{2}(\cdot)=b\left(Y_{2}(\cdot)\right)$. The Borel set $E=\{(x, y)$ : $b(x)=b(y)\} \subset \mathbb{R}^{2}$ is an equivalence relation. Let $A \in \mathcal{E}$ (that is, $A$ is a saturated Borel set), then $A=b^{-1}(b(A))$ and $b(A) \in \Sigma$. It is given that $P_{1}\left(X_{1} \in b(A)\right)=P_{2}\left(X_{2} \in\right.$ $b(A))$, that is, $P_{1}\left(Y_{1} \in A\right)=P_{2}\left(Y_{2} \in A\right)$. Denoting by $\mu_{1}, \mu_{2}$ the distributions of $Y_{1}, Y_{2}$ respectively, we see that $\mu_{1}(A)=\mu_{2}(A)$ for all $A \in \mathcal{E}$.

By Lemma 8.10, $I_{\mu_{1}, \mu_{2}}(E)=1$. By Theorem 3.4, $S_{\mu_{1}, \mu_{2}}(E)=1$. Lemma 8.12 gives us a probability measure $\mu$ on $\mathbb{R}^{2}$ with the marginals $\mu_{1}, \mu_{2}$ such that $\mu(E)=1$.

We consider the conditional distribution $P_{1, x}$ of $\omega_{1} \in \Omega_{1}$ given $Y_{1}\left(\omega_{1}\right)=x$ (its existence is ensured by standardness of $\left.\Omega_{1}\right) ; P_{1, x}$ is a probability measure on $\Omega_{1}$ for $\mu_{1}$-almost all $x \in \mathbb{R}$, and $\int_{\mathbb{R}} P_{1, x} \mu_{1}(\mathrm{~d} x)=P_{1}$. The same holds for $P_{2, y}$. We construct a probability measure $P$ on $\Omega_{1} \times \Omega_{2}$ by

$$
P=\int_{\mathbb{R}^{2}}\left(P_{1, x} \times P_{2, y}\right) \mu(\mathrm{d} x \mathrm{~d} y)
$$


The first marginal of $P$ is $\int P_{1, x} \mu(\mathrm{d} x \mathrm{~d} y)=\int P_{1, x} \mu_{1}(\mathrm{~d} x)=P_{1}$; the second marginal of $P$ is $P_{2}$. Also, $P\left(\left\{\left(\omega_{1}, \omega_{2}\right):\left(Y_{1}\left(\omega_{1}\right), Y_{2}\left(\omega_{2}\right)\right) \in E\right\}\right)=\mu(E)=1$. Thus, for $P$-almost all $\left(\omega_{1}, \omega_{2}\right)$ we have $\left(Y_{1}\left(\omega_{1}\right), Y_{2}\left(\omega_{2}\right)\right) \in E$, therefore, $b\left(Y_{1}\left(\omega_{1}\right)\right)=b\left(Y_{2}\left(\omega_{2}\right)\right)$, that is, $X_{1}\left(\omega_{1}\right)=$ $X_{2}\left(\omega_{2}\right)$.

8.13 Definition. Let $V$ be a borelogical space and $\Omega$ a standard probability space. Two $V$-valued random variables $X_{1}, X_{2}: \Omega \rightarrow V$ are independent, if there exist independent random variables $Y_{1}, Y_{2}: \Omega \rightarrow \mathbb{R}$ and a plot $b: \mathbb{R} \rightarrow V$ such that $X_{1}(\cdot)=b\left(Y_{1}(\cdot)\right)$ and $X_{2}(\cdot)=b\left(Y_{2}(\cdot)\right)$. Independence of three or more $V$-valued random variables is defined similarly, as well as independence of random elements of different borelogical spaces.

A given generating plot $b: \mathbb{R} \rightarrow V$ can be used always. Also, the case $X_{1}(\cdot)=b_{1}\left(Y_{1}(\cdot)\right)$, $X_{2}(\cdot)=b_{2}\left(Y_{2}(\cdot)\right)$ reduces to a single $b$.

If $X_{1}, X_{2}: \Omega \rightarrow V$ are independent then $\mathbb{P}\left(X_{1} \in A_{1}, X_{2} \in A_{2}\right)=\mathbb{P}\left(X_{1} \in A_{1}\right) \mathbb{P}\left(X_{2} \in\right.$ $\left.A_{2}\right)$ for all $A_{1}, A_{2} \in \Sigma$. The converse is generally wrong.

8.14 Counterexample. Let $X: \Omega \rightarrow \mathbb{R} / \mathbb{Q}$ have the absolutely continuous distribution (recall 8.5). Then $\mathbb{P}\left(X \in A_{1}, X \in A_{2}\right)=\mathbb{P}\left(X \in A_{1}\right) \mathbb{P}\left(X \in A_{2}\right)$ for all $A_{1}, A_{2} \in \Sigma$, but $X$ is not independent of itself.

Proof. The equality $\mathbb{P}\left(X \in A_{1}, X \in A_{2}\right)=\mathbb{P}\left(X \in A_{1}\right) \mathbb{P}\left(X \in A_{2}\right)$ is easy to check in each of the four possible cases $(0 \cdot 0,0 \cdot 1,1 \cdot 0,1 \cdot 1)$ taking into account that $X$ has a 0-1 distribution. (See also $[9,4.6]$.) Assume that $X=\mathbb{Q}+Y_{1}$ and $X=\mathbb{Q}+Y_{2}$ where $Y_{1}, Y_{2}: \Omega \rightarrow \mathbb{R}$ are independent. Then $\mathbb{P}\left(Y_{1}-Y_{2} \in \mathbb{Q}\right)=1$, therefore $\mathbb{P}\left(Y_{1}-Y_{2}=q\right)>0$ for some $q \in \mathbb{Q}$, and $0<\mathbb{P}\left(Y_{1}-Y_{2}=q \mid Y_{2}=y_{2}\right)=\mathbb{P}\left(Y_{1}=y_{2}+q\right)$ for some $y_{2}$; that is, $Y_{1}$ has an atom at $y_{1}=y_{2}+q$. On the other hand, $X_{1}=\mathbb{Q}+Y$ for some absolutely continuous $Y$. We have $\mathbb{P}\left(Y_{1}-Y \in \mathbb{Q}\right)=1$, therefore $\mathbb{P}\left(Y \in \mathbb{Q}+y_{1}\right)>0$ in contradiction to the absolute continuity of $Y$.

\section{Some generalizations and final remarks}

9.1 Remark. Theorem 6.1 remains true if 'the independence condition' is replaced with 'the quasi-independence condition' introduced as follows.

First, in Definition 4.1, instead of item (c) "the random sequence $\left(Y_{2}, Y_{4}, Y_{6}, \ldots\right)$ is independent of the random sequence $\left(Y_{1}, Y_{3}, Y_{5}, \ldots\right)$ " we write "the $\sigma$-fields $\sigma\left(Y_{1}, Y_{3}, Y_{5}, \ldots\right)$, $\sigma\left(Y_{2}, Y_{4}, Y_{6}, \ldots\right)$ are a nonsingular pair (on the whole probability space)", in which case we call the two fragments quasi-independent. Recall that 'nonsingular pair' means (according to Definition 5.5) absolute continuity of the joint distribution w.r.t. the product of its two marginals.

Second, in Definition 4.2, instead of "the $n$ fragments ... are independent" we write "the $n$ fragments ... are quasi-independent", thus defining the quasi-independence condition. 
The only change in the proof of Proposition 5.2 is, deletion of the last four words 'since they are independent'. The proof of Lemma 5.10 still holds. Now, Propositions $5.1,5.9$ and Theorem 6.1 use these modified 5.2 and 5.10 as before.

We see that quasi-independence is no less restrictive than independence, for random dense countable sets. For random closed sets the situation is completely different; independence characterizes Poisson processes, while quasi-independence leaves a great freedom [15, Sect. 6].

9.2 Remark. Returning to independence, we compare our approach with that of Kingman $\left[10\right.$, Sect. 2]. A Poisson process is defined there as a family $(X(\omega))_{\omega \in \Omega}$ of finite or countable subsets $X(\omega)$ of a given measurable space $T$, satisfying three conditions: measurability, independence and distribution. The measurability condition: for every measurable $B \subset T$ the number $\xi_{B}(\omega) \in\{0,1,2, \ldots\} \cup\{\infty\}$ of points in $B \cap X(\omega)$ is measurable in $\omega$. The independence condition: for any disjoint measurable $B_{1}, \ldots, B_{n} \subset T$ the random variables $\xi_{B_{1}}, \ldots, \xi_{B_{n}}$ are independent. The distribution condition: each random variable $\xi_{B}$ has the Poisson distribution with some parameter $\mu(B) \in[0, \infty]$; here $\mu(B)=0$ means that $\xi_{B}=0$ a.s., while $\mu(B)=\infty$ means that $\xi_{B}=\infty$ a.s.

Clearly, $\mu$ is a measure, positive, maybe infinite and even not $\sigma$-finite, and nonatomic in the sense that $\mu(\{t\})=0$ for all $t \in T$. It appears [10, Sect. 2.5] that such a Poisson process exists for every $\mu$ of the form $\mu_{1}+\mu_{2}+\ldots$ where $\mu_{n}$ are nonatomic finite positive measures on $T$.

For example, we may take $T=(0,1)$ and $\mu_{1}=\mu_{2}=\ldots$ be Lebesgue measure on $(0,1)$. Then Kingman's construction gives just the object $X$ that we call an unordered infinite sample. However, consider $Y(\omega)=(\mathbb{Q}+y(\omega)) \cap(0,1)$; here $\mathbb{Q} \subset \mathbb{R}$ is the set of all rational numbers, $\mathbb{Q}+y(\omega)$ its shift by $y(\omega)$, and $y: \Omega \rightarrow \mathbb{R}$ a random variable with an absolutely continuous distribution (as in 8.5, 8.14). Is $Y$ also a Poisson process?

In the framework of Kingman, $Y$ is a Poisson process, and moreover, $X$ and $Y$ are treated as identically distributed, just because random variables $\xi_{B}$ do not feel any difference between $X$ and $Y$. However, distances between points are irrational for $X$ but rational for $Y$; a clear-cut distinction!

In our framework (recall 2.2 and 8.3 ) $X$ and $Y$ are not identically distributed, and $Y$ should not be called a Poisson process, since it violates the independence condition 4.2 (similarly to 8.14).

See also [10, Sect. 2.2]: "It might be objected that $\Pi_{1}$ and $\Pi_{2}$ are not 'really' independent, and only appear to be so because we choose to describe them in terms of their count processes."

9.3 Counterexample. Kingman's measurability condition (mentioned in 9.2) does not imply the measurability condition (2.1). There exists a family $(X(\omega))_{\omega \in \Omega}$ of countable sets $X(\omega) \subset \mathbb{R}$ not of the form (2.1) but such that all $\xi_{B}$ are measurable. Here $\Omega$ is a standard probability space. 
We take $\Omega=(0,1)$ (with Lebesgue measure), choose an irrational number $a \in \mathbb{R}$ and a set $A \subset(0,1)$ of interior measure 0 but outer measure 1 , and define $X$ by

$$
X(\omega)= \begin{cases}\mathbb{Q}+\omega & \text { for } \omega \in A, \\ \mathbb{Q}+\omega+a & \text { for } \omega \in(0,1) \backslash A ;\end{cases}
$$

here $\mathbb{Q}$ is the set of all rational numbers, and $\mathbb{Q}+\omega$ its shift by $\omega$.

If a Borel set $B \subset \mathbb{R}$ is negligible (that is, of Lebesgue measure 0) then $\{\omega: X(\omega) \cap B \neq \emptyset\}$ is negligible (since it has negligible intersection with $A$ and also with $(0,1) \backslash A$ ); thus $\xi_{B}=0$ a.s. Otherwise, if $B$ is of positive measure, then $\{\omega: X(\omega) \cap B=\emptyset\}$ is negligible (for the same reason); it follows that $\xi_{B}=\infty$ a.s.

Assume that a function $X_{1}:(0,1) \rightarrow \mathbb{R}$ is such that $X_{1}(\omega) \in X(\omega)$ for almost all $\omega$. Then $X_{1}(\omega)-\omega$ is rational for almost all $\omega \in A$ but irrational for almost all $\omega \in(0,1) \backslash A$. Thus $X_{1}$ cannot be measurable, which shows that $X$ is not of the form (2.1).

Kingman's independence condition is too demanding for the percolation-related models mentioned in Introduction; independent fragments of their 'full scaling limit' are welldefined over disjoint good domains, not just Borel sets. An appropriate independence condition is given below.

Random finite or countable subsets of a standard Borel space $T$ are random elements (see 8.1) of the borelogical space FCS $(T)$ defined similarly to 7.8. They may be written as $X=\left\{X_{1}, \ldots, X_{N}\right\}$ where $X_{1}, X_{2}, \cdots: \Omega \rightarrow T$ and $N: \Omega \rightarrow\{0,1,2, \ldots\} \cup\{\infty\}$ are random variables. Here $X(\omega)=\left\{X_{1}(\omega), \ldots, X_{N(\omega)}(\omega)\right\}$ when $N(\omega)<\infty$ and $X(\omega)=$ $\left\{X_{1}(\omega), X_{2}(\omega), \ldots\right\}$ when $N(\omega)=\infty$. Of course, $N(\omega)=0$ means $X(\omega)=\emptyset$.

Given a Borel set $B \subset T$, the fragment $\omega \mapsto B \cap X(\omega)$ of $X$ is again a random finite or countable set. Independence of two or more such fragments is understood according to 8.13. It is less clear how to generalize 4.2 since it is based on intervals.

9.4 Definition. Let $T$ be a standard Borel space, $X$ a random finite or countable subset of $T$, and $\mathcal{A}$ an algebra of Borel subsets of $T$. We say that $X$ satisfies the independence condition on $\mathcal{A}$, if for every $n=2,3, \ldots$ and every $n$ disjoint sets $B_{1}, \ldots, B_{n} \in \mathcal{A}$, the $n$ fragments $B_{1} \cap X, \ldots, B_{n} \cap X$ are independent.

By the way, a countable algebra $\mathcal{A}$ generates the Borel $\sigma$-field if and only if it separates points (see [7, (14.16)]).

9.5 Lemma. If $X$ satisfies the independence condition on some algebra $\mathcal{A}$ that generates the Borel $\sigma$-field of $T$, and $\mathbb{P}(t \in X)=0$ for all $t \in T$, then $X$ is a Poisson process in the sense of Kingman (see 9.2).

Proof. The three conditions mentioned in 9.2 must be verified. The measurability condition holds evidently. The independence condition evidently holds for $B_{1}, \ldots, B_{n} \in \mathcal{A}$; after some preparations it will be generalized to all measurable $B_{1}, \ldots, B_{n}$. 
There exists a nonatomic finite positive measure $\nu$ on $T$ such that

$$
\nu(B)=0 \quad \text { if and only if } \mathbb{P}(B \cap X \neq \emptyset)=0
$$

for all Borel $B \subset T$. For example, one may represent $X$ as $\left\{X_{1}, \ldots, X_{N}\right\}$ and take $\nu(B)=\sum_{n} 2^{-n} \mathbb{P}\left(N \geq n, X_{n} \in B\right)$.

For every Borel $B \subset T$ there exist $A_{1}, A_{2}, \cdots \in \mathcal{A}$ such that $\nu\left(B \triangle A_{n}\right) \rightarrow 0$ and moreover, $\sum_{n} \nu\left(B \triangle A_{n}\right)<\infty$. (Of course, $B \triangle A_{n}=\left(B \backslash A_{n}\right) \cup\left(A_{n} \backslash B\right)$.) We introduce sets $C_{n}=\bigcap_{k>n} A_{k}, C=\bigcup_{n} C_{n}$ and $D_{n}=\bigcap_{k>n}\left(T \backslash A_{k}\right), D=\bigcup_{n} D_{n}$, then $C_{n} \uparrow C, D_{n} \uparrow D$, $C \cap D=\emptyset$ and $\nu(B \backslash C)=0, \nu((T \backslash B) \backslash \bar{D})=0$ by the first Borel-Cantelli lemma. Thus, $\nu(B \triangle C)=0$ and $\nu((T \backslash B) \triangle D)=0$.

For each $n$ we have $C_{n} \subset A_{n}$ and $D_{n} \subset T \backslash A_{n}$; the independence condition on $\mathcal{A}$ implies that $\xi_{C_{n}}, \xi_{D_{n}}$ are independent. However, $\xi_{C_{n}} \uparrow \xi_{C}=\xi_{B}$ and $\xi_{D_{n}} \uparrow \xi_{D}=\xi_{T \backslash B}$; we conclude that $\xi_{B}$ and $\xi_{T \backslash B}$ are independent for every Borel set $B \subset T$. Similarly, $\xi_{B_{1}}, \ldots, \xi_{B_{n}}$ are independent whenever Borel sets $B_{1}, \ldots, B_{n}$ are disjoint (this generalization is left to the reader); the independence condition is verified.

Without loss of generality we assume that $T=(0,1)$ and $\nu$ is absolutely continuous w.r.t. Lebesgue measure (by the isomorphism theorem for measure spaces, see [7, (17.41)] or [13, 3.4.23]). We cannot apply Prop. 4.4 'as is', since the algebra $\mathcal{A}$ need not contain intervals (essential in 4.2). However, the independence condition 4.2 is used in the proof of 4.4 only once; it ensures independence of the random variables $\xi(x, y)$ for disjoint intervals $(x, y)$. In our case, independence between these $\xi(x, y)$ is ensured by the independence condition formulated in 9.2 and verified above. Thus, the conclusion of Prop. 4.4 holds; the distribution condition is verified.

9.6 Remark. Let $X$ be as in Lemma 9.5, then $\mu$ defined by $\xi_{B} \sim \operatorname{Poisson}(\mu(B))$ is a measure of the form $\mu_{1}+\mu_{2}+\ldots$ where $\mu_{n}$ are nonatomic finite positive measures on $T$. Indeed, representing $X$ as $\left\{X_{1}, \ldots, X_{N}\right\}$ we have $\mu(B)=\sum_{n} \mathbb{P}\left(N \geq n, X_{n} \in B\right)$.

On the other hand, every such measure $\mu$ corresponds to some $X$, which follows from Kingman's construction (mentioned in 9.2).

9.7 Remark. The independence condition 4.2 may be treated as a special case of the independence condition 9.4 ; namely, $T=(0,1)$ and $\mathcal{A}$ consists of finite unions of intervals. Similarly we may take $T=\mathbb{R}^{2}$ and $\mathcal{A}$ consisting of finite unions of rectangles. Using 9.5 we may generalize 4.4 to the two-dimensional case. This way, the main results (Theorems 6.1, 6.9) may be generalized to dimension 2 (and higher).

9.8 Counterexample. It may happen that the distribution of the sequence $\left(X_{2}, X_{1}, X_{4}, X_{3}, X_{6}, X_{5}, \ldots\right)$ satisfies the conditions of Main lemma 1.2, but the distribution of the sequence $\left(X_{1}, X_{2}, X_{3}, \ldots\right)$ does not.

We construct random variables $X_{k}$ via their binary digits $\beta_{k, l}: \Omega \rightarrow\{0,1\}$,

$$
X_{k}=\left(0 . \beta_{k, 1} \beta_{k, 2} \ldots\right)_{2}=\sum_{l} 2^{-l} \beta_{k, l} .
$$


Each $\beta_{k, l}$ takes on the two values 0,1 with the probabilities $\frac{1}{2}, \frac{1}{2}$ and they all are independent except for the following restriction:

$$
\beta_{k+1, l}=\beta_{k, k+l-1} \quad \text { for } l=1, \ldots, k
$$

and $k=1,2, \ldots$

The conditional distribution of $X_{n+1}$ given $X_{1}, \ldots, X_{n}$ is the uniform distribution on an interval of length $2^{-n}$. Thus, Condition 1.2(a) is satisfied, but 1.2(b) is violated; moreover, the series of $1.2(\mathrm{~b})$ converges almost everywhere.

Condition 1.2(a), being permutation-invariant, is still satisfied by the distribution of $\left(X_{2}, X_{1}, X_{4}, X_{3}, X_{6}, X_{5}, \ldots\right)$. Condition 1.2(b) is also satisfied, since $X_{2 n}$ is independent of $X_{1}, \ldots, X_{2 n-2}$, which makes every second term of the series equal to 1 almost everywhere.

9.9 Remark. Let $\mu_{1}, \mu_{2}$ be two different probability measures on $(0,1)$, equivalent (that is, mutually absolutely continuous) to Lebesgue measure. Consider the mixture $\nu=$ $\frac{1}{2}\left(\mu_{1}^{\infty}+\mu_{2}^{\infty}\right)$ of the corresponding product measures $\mu_{1}^{\infty}, \mu_{2}^{\infty}$ on $(0,1)^{\infty}$. The measure $\nu$ is invariant under the group $S^{\infty}$ of all permutations, that is, invertible maps $s:\{1,2, \ldots\} \rightarrow$ $\{1,2, \ldots\}\left(S^{\infty}\right.$ acts on $(0,1)^{\infty}$ by $s x=\left(x_{s(1)}, x_{s(2)}, \ldots\right)$ for $\left.x=\left(x_{1}, x_{2}, \ldots\right)\right)$. Consider also the countable subgroup $S_{\infty} \subset S^{\infty}$ consisting of $s$ such that the set $\{n: s(n) \neq n\}$ is finite.

There exists an $S_{\infty}$-invariant Borel set $B \subset(0,1)^{\infty}$ such that $\mu_{1}^{\infty}(B)=1$ but $\mu_{2}^{\infty}(B)=0$. For example we may choose a Borel $A \subset(0,1)$ such that $\mu_{1}(A)<\mu_{2}(A)$ and take

$$
B=\left\{\left(x_{1}, x_{2}, \ldots\right): \limsup _{n \rightarrow \infty} \frac{1}{n}\left(\mathbf{1}_{A}\left(x_{1}\right)+\cdots+\mathbf{1}_{A}\left(x_{n}\right)\right)<\mu_{2}(A)\right\} .
$$

The set $B$ is not $S^{\infty}$-invariant, however, it is $S^{\infty}$-invariant mod 0 , that is, $\nu(B \triangle s B)=0$ for each $s \in S^{\infty}$.

In contrast, $\mu_{1}^{\infty}(B)=\mu_{2}^{\infty}(B)$ for every $S^{\infty}$-invariant Borel set $B \subset(0,1)^{\infty}$. Proof. Both $\mu_{1}^{\infty}$ and $\mu_{2}^{\infty}$ satisfy the conditions of Main lemma 1.2, therefore they lead to the same distribution on $\operatorname{CS}(0,1)$ (recall 2.4 ).

9.10 Remark. Finite or countable sets may be treated as equivalence classes of discrete probability measures (equivalence being mutual absolute continuity), see 7.8. Equivalence classes of nonatomic singular measures are another borelogical space. Random elements of this space may be subjected to conditions of independence and stationarity. An interesting example associated with Brownian motion is discussed in [14, Sect. 2f] in connection with a nonclassical noise (Warren's noise of stickiness).

9.11 Remark. Independence and stationarity of Brownian local minimizers result from (a) independence and stationarity of Brownian increments (the white noise) and (b) factorizability and stationarity of the map from Brownian increments to Brownian local minimizers. In terms of [14, Sect. 2e] this map is an example of a stationary local random dense countable set over the white noise. Brownian local maximizers are another 
example. Their union, Brownian local extrema, are the third example. (Several types of special points on the Brownian path are examined, see [11], but they are uncountable sets.) The question [14, 2e3], whether or not these three examples exhaust all stationary local random dense countable sets over the white noise, is still open!

\section{References}

[1] D.J. Aldous, M.T. Barlow (1981): On countable dense random sets, Lect. Notes Math. 850, 311-327. MR83f:60076

[2] F. Camia, L.R.G. Fontes, C.M. Newman (2005): The scaling limit geometry of near-critical $2 D$ percolation, arXiv:cond-mat/0510740v1.

[3] L.R.G. Fontes, M. Isopi, C.M. Newman, K. Ravishankar (2003): The Brownian web: characterization and convergence, arXiv:math.PR/0304119v1.

[4] P. Iglesias-Zemmour, Diffeology (preliminary draft, July 2005, unpublished). http://www. umpa.ens-lyon.fr/〜iglesias/

[5] I. Karatzas, S.E. Shreve (1991): Brownian motion and stochastic calculus, Springer (second edition). MR92h:60127

[6] A.S. Kechris (1999): New directions in descriptive set theory, The Bulletin of Symbolic Logic 5:2, 161-174. MR2001h:03090

[7] A.S. Kechris (1995): Classical descriptive set theory, Graduate Texts in Math. 156, Springer. MR96e:03057

[8] H.G. Kellerer (1984): Duality theorems for marginal problems, Z.Wahrscheinlichkeitstheorie verw. Gebiete 67:4, 399-432. MR86i:28010

[9] W.S. Kendall (2000): Stationary countable dense random sets, Adv. in Appl. Probab. 32, 86-100. MR2001g:60024

[10] J.F.C. Kingman (1993): Poisson processes, Clarendon, Oxford. MR94a:60052

[11] Y. Peres (2001): An invitation to sample paths of Brownian motion, lecture notes (unpublished). http://stat-www. berkeley.edu/pub/users/peres/bmall.pdf

[12] R. Pinciroli (2005): Countable Borel equivalence relations and quotient Borel spaces, arXiv:math.LO/0512626v1.

[13] S.M. Srivastava (1998): A course on Borel sets, Springer. MR99d:04002

[14] B. Tsirelson (2004): Nonclassical stochastic flows and continuous products, Probability Surveys 1, 173-298. MR2005m:60080 
[15] B. Tsirelson (2003): Non-isomorphic product systems, In: Advances in Quantum Dynamics (eds. G. Price et al), Contemporary Mathematics 335, AMS, pp. 273-328. MR2005b:46149 (Also arXiv:math.FA/0210457v2.) 